\title{
Ageostrophic instability in rotating, stratified interior vertical shear flows
}

\author{
Peng Wang ${ }^{a},{ }^{*}$, James C. McWilliams ${ }^{a}$ and Claire Ménesguen ${ }^{b}$ \\ a Department of Atmospheric and Oceanic Sciences, University of California, Los Angeles, CA 90095-1565, USA \\ ${ }^{\mathrm{b}}$ Department of Marine Geosciences, IFREMER, 29280 Plouzané, France \\ *: Corresponding author : Peng Wang, email address : pengwang@epss.ucla.edu
}

\begin{abstract}
:
The linear instability of several rotating, stably stratified, interior vertical shear flows $\bar{U}(z)$ is calculated in Boussinesq equations. Two types of baroclinic, ageostrophic instability, Al1 and Al2, are found in odd-symmetric $\bar{U}(z)$ for intermediate Rossby number $(R o)$. Al1 has zero frequency; it appears in a continuous transformation of the unstable mode properties between classic baroclinic instability $(\mathrm{BCl})$ and centrifugal instability $(\mathrm{Cl})$. It begins to occur at intermediate $R o$ values and horizontal wavenumbers $(k, l)$ that are far from $l=0$ or $k=0$, where the growth rate of $\mathrm{BCl}$ or $\mathrm{Cl}$ is the strongest. Al1 grows by drawing kinetic energy from the mean flow, and the perturbation converts kinetic energy to potential energy. The instability AI2 has inertia critical layers (ICL); hence it is associated with inertiagravity waves. For an unstable Al2 mode, the coupling is either between an interior balanced shear wave and an inertia-gravity wave (BG), or between two inertia-gravity waves (GG). The main energy source for an unstable BG mode is the mean kinetic energy, while the main energy source for an unstable GG mode is the mean available potential energy. Al1 and BG type Al2 occur in the neighbourhood of $A-S=0$ (a sign change in the difference between absolute vertical vorticity and horizontal strain rate in isentropic coordinates; see McWilliams et al., Phys. Fluids, vol. 10, 1998, pp. 3178-3184), while GG type Al2 arises beyond this condition. Both Al1 and Al2 are unbalanced instabilities; they serve as an initiation of a possible local route for the loss of balance in 3D interior flows, leading to an efficient energy transfer to small scales.
\end{abstract}

Keywords: baroclinic flows ; Instability ; waves in rotating fluids

\section{Introduction}

The general circulation of the ocean and atmosphere is constrained by approximately "balanced" dynamics, i.e., mostly geostrophic in horizontal momentum and hydrostatic in vertical momentum. The circulation is strongly influenced by rotation and stable stratification, with a small Rossby number $R o=$ $U_{0} / f L$ and Froude number $F r=U_{0} / N H$, where $U_{0}$ is the characteristic velocity scale, $f$ and $N$ are Coriolis and buoyancy frequencies, and $L(H)$ is the horizontal (vertical) length scale. The ratio of $R o$ and $F r$ defines a Burger number $B u=\mathrm{Ro}^{2} / \mathrm{Fr}^{2}=(N H)^{2} /(f L)^{2}$, which represents the square of the ratio between the deformation radius $\mathrm{NH}$ /f and the horizontal length scale of the flow. Mesoscale eddies in the ocean or synoptic scale storms in the atmosphere have typically small $R o$ and $F r$, with $B u$ around 1 ; they are approximately balanced motions. The 
balanced dynamics belongs to the "slow manifold" of advection and potential vorticity dynamics, which are manifested in an approximate model called Balanced Equations. As $R o \rightarrow 0$ with $B u \sim 1$, quasigeostrophic equations are asymptotically accurate as Balanced Equations. For finite Ro, the Balanced Equations manifest a higher-order momentum balance (e.g., gradient-wind balance) (Lorenz 1960; Charney 1962). In contrast, the "fast manifold", which is excluded from the balanced dynamics, is categorized as "unbalanced" motions, whose primary paradigm is inertia-gravity waves. Because there is no exact separation between the "slow manifold" and "fast manifold", the "slow manifold" is often claimed to be "fuzzy": though the Balanced Equations accurately describe the dynamics of the "slow" component of the flow, they do not evolve purely without a "fast" component that is often extremely small when Ro is small (Lorenz 1986; Lorenz \& Krishnamurthy 1987; Warn 1997).

The majority of kinetic and available potential energy in the general circulation is maintained at the planetary and mesoscale (or synoptic) scales; on the other hand, the kinetic energy dissipation is only effective on the molecular scale. For an energy input by planetary-scale forcing, how is the energy transferred towards small scales and finally dissipated? The nonlinear dynamics in balanced flows, i.e., geostrophic turbulence, is understood to be a very inefficient route due to its "inverse cascade" (Charney 1971). Therefore, breakdown of balance, manifested as an energy transfer to unbalanced motions, can be an important route for a forward turbulent energy cascade and dissipation. The boundary regions (e.g., turbulent boundary layer, bottom topography, and coast) are generally known as places where the flows are unbalanced and dissipation occurs. A local route to breakdown the balance in the interior ocean or atmosphere is not yet generally understood. The classic barotropic (Drazin \& Reid 1981) and baroclinic (Charney 1947; Eady 1949; Phillips 1954) instabilities, which are associated with an inflection point in the velocity profile (where potential vorticity gradient changes sign), occur within the constraints of balance, hence are not likely to be a route for the breakdown of balance. The purpose of this study is to look for possible types of ageostrophic instability that can provide a local route to a breakdown of balanced flows in the interior.

McWilliams et al. (1998) proposed three sufficient conditions for the occurrence of the breakdown of balanced evolution in a 3D stratified flow; they are: 1) sign change of stratification $N^{2}$, which is related to gravitational instability (Chandrasekhar 1961); 2) sign change of the absolute vorticity in isentropic coordinates $A$ (proportional to Ertel potential vorticity, $P V$ ), which is related to centrifugal instability (Ooyama 1966); 3) sign change of $A^{2}-S^{2}$, where $S$ is the magnitude of the horizontal strain rate in isentropic coordinates. (The formulas for $A, S$ and $P V$ are in Sec. 2.) For $f>0$ sign change means from positive to negative. If we consider a flow that is centrifugally stable $(A>0)$, the third condition $A^{2}-S^{2}=(A+S)(A-S)$ becomes sign change of $A-S$. With $S$ always positive, it can be satisfied more commonly than the first two conditions with a moderate but finite $R o$. Because $A$ is smaller in anticyclonic flows, the instabilities that are relevant to the $A-S$ condition are often called anticyclonic, ageostrophic instability (AAI). Unlike the other two criteria for loss of balance $\left(N^{2}<0\right.$ and $\left.A<0\right)$ that are associated with known instability types with sharp onset conditions (gravitational and centrifugal, respectively), the occurrence of AAI may be in the neighborhood of, rather than precisely at, $A-S=0$. In addition to the "fuzziness" of the "slow manifold", Balanced Equations are not uniquely defined historically. There are several different Balanced Equations that accurately describe "slow" dynamics (Leith 1980; Lorenz 1980; Gent \& McWilliams 1982; Warn et al. 1995). The conditions for loss of balance in McWilliams et al. (1998) are based on a particular set of Balanced Equations in isentropic coordinates that are analogous to the height-coordinate formulation in Lorenz (1960). Therefore the $A-S<0$ criterion 
is not uniquely defined, and other Balanced Equations may have a similar criterion nearby but not exactly the same. Besides the three types of ageostrophic instability that are associated with the above three sufficient conditions, there are other types, such as Kelvin-Helmholtz instability (Miles 1961), which occurs when Richardson number $R i=N^{2} / U_{z}^{2}<\frac{1}{4}$ in non-rotating flows.

The previous literature has identified several types of ageostrophic instabilities. In rotating shallow water flows (Griffiths et al. 1982; Paldor 1983; Kubokawa 1985, 1986; Hayashi \& Young 1987; Dritschel \& Vanneste 2006; Gula \& Zeitlin 2010; Wang et al. 2012 ) and two-layer hydrostatic frontal flows with both barotropic and baroclinic shears (Killworth et al. 1984; Boss et al. 1996; Gula et al. 2010), the ageostrophic instabilities are identified as a resonance between a balanced wave (shear wave supported by potential vorticity gradient or frontal wave) and an unbalanced wave (inertia-gravity wave or Kelvin wave), or between two unbalanced waves. Bouchut et al. (2011) examine the barotropic Bickley jet in a two-layer shallow-water model and find a continuous transformation among barotropic instability, symmetric centrifugal instability and asymmetric centrifugal instability as Ro increases. Sakai (1989), Sutyrin (2007), and Gula et al. (2009) find a type of baroclinic, ageostrophic instability as a resonance between a shear wave (Rossby wave) and a Kelvin wave in a channeled, two-layer shallow water model. Several 3D instability problems have been solved in a stratified barotropic flow (McWilliams \& Yavneh 1998; Yavneh et al. 2001; Molemaker et al. 2001; McWilliams et al. 2004; Vanneste \& Yavneh 2007), where the ageostrophic instabilities are identified as a type of AAI, and their growth rate has an inverse exponential dependence on Ro. Ménesguen et al. (2012) studied a stratified, interior 2D jet with both barotropic and baroclinic shear, and found the characteristics of the unstable modes are transformed through a near-degeneracy in the neighborhood of $A-S=0$. The ageostrophic instability in an Eady flow is well understood from earlier studies (Stone 1966; Nakamura 1988; Molemaker et al. 2005; Plougonven et al. 2005). It is interpreted as a coupling between an edge Rossby wave and a Doppler-shifted inertia-gravity wave. Its growth rate strongly increases near the neighborhood of $A-S=0$, hence it is also identified as a type of AAI. An ageostrophic instability which couples topographic Rossby waves and inertia-gravity waves is found by Sutyrin (2008) in a stratified layer of fluid between two homogenous layers, with constant $A$ and $S$, and $A-S \neq 0$.

In most of the examined cases, the occurrence of an ageostrophic instability relies on the existence of boundaries, equator, vanishing layer depth, or singular potential vorticity. Our work aims to understand the generic characteristic of the ageostrophic instability in a continuously differentiable interior flow $\bar{U}(z)$. If we review the criteria of currently known linear instability conditions with increasing $R o$, the instabilities appear in the following order: baroclinic instability (BCI), anticyclonic, ageostrophic instability (AAI), centrifugal instability (CI), Kelvin-Helmholtz instability (KHI), and finally gravitational instability (GI) when the flow is unstably stratified. Our goal is to understand a general local route for the loss of balance within the control of rotation and stable stratification, so we are more interested in the ageostrophic instabilities that occur for an intermediate Ro (below the criteria for CI, KHI and GI). The occurrence of ageostrophic instability in shallow-water or layered hydrostatic flows requires a sharp condition: $\mathrm{Fr}$ exceeds a critical value (related to the Ripa (1983) second stability condition). In contrast, the studies in continuously stratified flows all show that the ageostrophic instabilities have a growth rate without a sharp cutoff in $R o$ (due to the lack of lower bound of both the vertical scale and horizontal inertia-gravity wave phase speeds in stratified flows); and some of the instabilities imply a coupling with inertia-gravity waves. Most of the examined, different types of ageostrophic instabilities in different flows are associated with the $A-S$ 
condition. In this paper we focus on the linear instabilities of a rotating, stably stratified (constant $N^{2}$ ), interior baroclinic shear flow $\bar{U}(z)$ with non-uniform potential vorticity in Boussinesq equations. The following questions are addressed: (i) What are the generic types of ageostrophic instability in a rotating, stably stratified, interior baroclinic shear flow with $A>0$ ? (ii) Does the instability occur or become strong in the neighborhood of $A-S=0$ ? Does it always relate to the coupling of the mean shear and inertia-gravity waves? The rest of the paper is constructed as follows: Section 2 specifies the equations, the non-dimensional parameters, and the mean flows. A brief explanation of the methods that we use to diagnose the unstable modes is in Sec. 3. The first type of ageostrophic instability (AI1), a continuous transformation between BCI and CI, is described in Sec. 4, followed by Sec. 5 for the second type of ageostrophic instability (AI2) that is associated with inertia critical layers (ICL). The paper summarizes with a discussion in Sec. 6 .

\section{Equations, parameters and mean flows}

We solve the instability problem with a $f$-plane, constant-stratification $\left(N^{2}=\right.$ constant), linearized Boussinesq equations. To non-dimensionalize the variables, we scale them as follows:

$$
\begin{aligned}
& (x, y) \rightarrow L, \quad z \rightarrow H, \quad(u, v) \rightarrow U_{0}, \quad t \rightarrow \frac{L}{U_{0}}, \\
& w \rightarrow W_{0}=\frac{f U_{0}^{2}}{N^{2} H}, \quad p \rightarrow f U_{0} \rho_{0} L, \quad b \rightarrow \frac{f U_{0} L}{H},
\end{aligned}
$$

where $u$ and $v(w)$ are the horizontal (vertical) velocities, $p$ is the pressure field, $b=$ $-g \rho / \rho_{0}$ is the buoyancy field, and $\rho_{0}$ is the average density. For a small perturbation $\left\{u^{\prime}\right.$, $\left.v^{\prime}, w^{\prime}, p^{\prime}, b^{\prime}\right\}$ around a thermal-wind balanced mean state $\{\bar{U}(z), 0,0, \bar{P}(y, z), \bar{B}(y, z)\}$, the linearized non-dimensional Boussinesq equations are

$$
\left\{\begin{aligned}
R o\left(\frac{\partial u^{\prime}}{\partial t}+\bar{U} \frac{\partial u^{\prime}}{\partial x}+\frac{R o}{B u} \bar{U}_{z} w^{\prime}\right)-v^{\prime}+\frac{\partial p^{\prime}}{\partial x} & =0 \\
R o\left(\frac{\partial v^{\prime}}{\partial t}+\bar{U} \frac{\partial v^{\prime}}{\partial x}\right)+u^{\prime}+\frac{\partial p^{\prime}}{\partial y} & =0 \\
\lambda^{2} \frac{R o^{2}}{B u}\left(\frac{\partial w^{\prime}}{\partial t}+\bar{U} \frac{\partial w^{\prime}}{\partial x}\right)-b^{\prime}+\frac{\partial p^{\prime}}{\partial z} & =0 \\
\frac{\partial u^{\prime}}{\partial x}+\frac{\partial v^{\prime}}{\partial y}+\frac{R o}{B u} \frac{\partial w^{\prime}}{\partial z} & =0 \\
\frac{\partial b^{\prime}}{\partial t}+\bar{U} \frac{\partial b^{\prime}}{\partial x}+\bar{B}_{y} v^{\prime}+\frac{R o}{B u} \bar{B}_{z} w^{\prime} & =0 .
\end{aligned}\right.
$$

There are three important non-dimensional parameters in the equations: Rossby number $R o$, Burger number $B u$, and aspect ratio $\lambda$,

$$
R o=\frac{U_{0}}{f L}, \quad B u=\left(\frac{N H}{f L}\right)^{2}, \quad \lambda=\frac{H}{L} .
$$

The Froude number can be defined by $R o$ and $B u: F r=\frac{U_{0}}{N H}=\frac{R o}{\sqrt{B} u}$. Because the mean flow velocity $\vec{V}(z)=(\bar{U}(z), 0,0)$ is uniform in the horizontal plane, the horizontal length scale $L$ is arbitrary. Therefore, the three parameters $(R o, B u, \lambda)$ are not independent of each other. We choose $B u=1$ (a typical value for mesoscale eddies or synoptic storms), and then $R o$ and $\lambda$ become free parameters. The non-dimensional translations between equations with $(B u, R o, \lambda)$ and those with the equivalent combinations $\left(B u=1, R o^{*}\right.$, 
$\left.\lambda^{*}\right)$ are discussed in Appendix A. For the large-scale or mesoscale (synoptic-scale) flows, the horizontal scale is much larger than the vertical scale, so we choose a small value of aspect ratio ( $\lambda=0.01$ in our study). We look for the ageostrophic instability in a balanced flow for intermediate $R o$. The mean flow satisfies thermal-wind balance $\bar{B}_{y}=-\bar{U}_{z}$, thus

$$
\bar{B}(y, z)=\frac{B u}{R o} z-\bar{U}_{z} y .
$$

Here the first term in $\bar{B}$ is the background stratification, and the second term represents the horizontal buoyancy slope induced by the thermal-wind balanced vertical shear. The vertical gradient of buoyancy is

$$
\bar{B}_{z}=\frac{B u}{R o}-\bar{U}_{z z} y
$$

If $R o$ and the meridional scale of the perturbation are not large, the first term in (2.4) is dominant over the second. We consider solutions with small and intermediate Ro or meridional scale, so the second term in $\bar{B}_{z}$ is dropped in the buoyancy equation by solving the problem in the neighborhood of $y=0$. This allows a solution with a $\mathrm{e}^{i l y}$ form in the meridional direction. With this simplification, we assume

$$
\left\{u^{\prime}, v^{\prime}, w^{\prime}, p^{\prime}, b^{\prime}\right\}(x, y, z, t) \sim \operatorname{Re}\left[\{\hat{u}, \hat{v}, \hat{w}, \hat{p}, \hat{b}\}(z) e^{i(k x+l y)} e^{\tilde{\sigma} t}\right],
$$

where $\sigma=\operatorname{Real}(\tilde{\sigma})$ is the growth rate, and we use $\omega=-\operatorname{Imag}(\tilde{\sigma})$ to denote frequency, then (2.1) becomes a $1 \mathrm{D}$ eigenvalue problem in $z$ :

$$
\left\{\begin{aligned}
R o\left(\tilde{\sigma} \hat{u}+i k \bar{U} \hat{u}+\frac{R o}{B u} \bar{U}_{z} \hat{w}\right)-\hat{v}+i k \hat{p} & =0 \\
R o(\tilde{\sigma} \hat{v}+i k \bar{U} \hat{v})+\hat{u}+i l \hat{p} & =0 \\
\lambda^{2} \frac{R o^{2}}{B u}(\tilde{\sigma} \hat{w}+i k \bar{U} \hat{w})-\hat{b}+\frac{\partial \hat{p}}{\partial z} & =0 \\
i k \hat{u}+i l \hat{v}+\frac{R o}{B u} \frac{\partial \hat{w}}{\partial z} & =0 \\
\tilde{\sigma} \hat{b}+i k \bar{U} \hat{b}-\bar{U}_{z} \hat{v}+\hat{w} & =0 .
\end{aligned}\right.
$$

The above approximation that the solution has a $\mathrm{e}^{i l y}$ form in $y$ is valid when the meridional variation of the mean flow is small around $y=0$ compared to perturbation scale; i.e., there is a meridional scale separation between the mean state and the perturbations, which is true if $R o$ is not large or $l$ is not small according to (2.4). If the vertical shear induced horizontal buoyancy variation is small enough (small Ro), or the perturbation scale is small enough (large $l$ ), the second term in (2.4) is negligible. We call it "wide jet approximation".

For instance, most calculations for ageostrophic instabilities in our study for $R o<1$ are safe with this approximation. When $R o$ is close to 1 , the solutions to (2.5) with small $l$ might not be exactly the solutions to (2.1) because the two terms in (2.4) are comparable. Our focus is the ageostrophic instabilities that are usually in small scales (with large $l$ ), therefore the discrepancy in large $R o$ and small $l$ cases would not affect our main conclusions. To be careful, we have performed a reliability check on all the solutions with examined $(R o, l)$ values by comparing the local magnitude of the two terms in (2.4) as a function of $z$. For perturbations with a meridional wavenumber $l$, the following condition should be satisfied to make the "wide jet approximation" valid:

$$
\frac{B u}{R o}>\max \left[\left|\bar{U}_{z z}\right|\right] / l,
$$


where $B u$ is 1 . Therefore, for a fixed $R o$, there is a lower limit of $l=R o \cdot \max \left[\left|\bar{U}_{z z}\right|\right]$ for the solutions to be reliable. This safety check is performed for all the results shown in this manuscript. The result of the reliability check for the solutions with this approximation is specified as they are shown in Sec. 4 and 5 .

An general way to calculate the exact solutions to $(2.1)$ is to solve a $2 \mathrm{D}$ eigenvalue problem in a $y$-bounded domain, with eigenfunctions that are non-seperatable in $y$ and $z$. However, accurate calculations of the small-scale perturbations require very high resolution (a few thousands in each dimension), hence they are computationally demanding (as reported in Ménesguen et al. (2012)). Our problem could be revisited by calculating the fully $2 \mathrm{D}$ eigenfunctions in the future when computational power is improved.

We solve this system for small aspect ratio $(\lambda=0.01)$ and $R o$ increasing from 0 to values beyond the onset condition for CI, but below that for KHI. The problem is solved by a Fortran code with ARPACK library (an eigensolver library). Because a large resolution is needed for an accurate solution, it is too expensive to compute the full-spectrum of eigenvalues. ARPACK computes a few eigenvalues and corresponding eigenfunctions close to the given "target" (guessed eigenvalue) by an iterative convergence process. The differential operators are discretized by a second-order centered finite-difference method, in a staggered grid, with a resolution of $N \sim 5000$ in the vertical direction. We do not look for radiating instabilities, which might exist as solutions to (2.5) by setting a propagating boundary condition, therefore none of the unstable modes found by our eigensolver has a vertical propagation. We make sure the domain size in the computation is sufficiently large to remove the boundary influence on the unstable modes in the interior shear flows. For simplicity, we choose a rigid and no-flux boundary condition set comprised of $w^{\prime}=0$ and $\partial u^{\prime} / \partial z=\partial v^{\prime} / \partial z=\partial p^{\prime} / \partial z=\partial b^{\prime} / \partial z=0$.

Some of our ageostrophic modes involve inertia-gravity waves and have inertia critical layers in the vertical structure. To accurately determine the growth rate and resolve the mode structure, we solve (2.5) with very small viscous and diffusive terms. On the right hand sides of zonal, meridional momentum and buoyancy equations in (2.5), we add $E k \nabla^{2} \hat{u}, E k \nabla^{2} \hat{v}$, and $\frac{E k}{P r} \nabla^{2} \hat{b}$, respectively, where $E k=\nu /\left(f L^{2}\right)$ is the Ekman number, and $\operatorname{Pr}=\nu / \kappa$ is the Prandtl number, with $\nu$ and $\kappa$ the kinetic viscosity and thermal diffusion. For simplicity, we choose $\operatorname{Pr}=1$. We have tested the numerical results with vanishing $E k$ values and make sure the viscosity and diffusion are small enough so that they have negligible effect on the unstable modes. The ageostrophic modes that are not associated with inertia critical layers can be well determined without viscosity and diffusion terms. On the other hand, we have increased the grid resolution from 3000 to 10000 , and have confirmed that the eigenvalue converges well with $N=5000$ for the unstable modes of interest. The correctness of our numerical solver is confirmed by reproducing the results for Eady AI mode (which has an inertia critical layer in the mode structure) in Stone (1970) (Fig. 3 and 4) and Molemaker et al. (2005) (Fig. 1, 2, 6 and 7).

Two types of interior vertical shear flows are examined: the hyperbolic tangent flow (TANH) and the double-jet flow (DJET). Although the EADY flow has been well studied by earlier literature, we revisit it for comparison with the TANH and DJET flows. The analytical forms for the mean flows $\bar{U}(z)$ are

EADY $: \bar{U}=z$, TANH $: \bar{U}=0.5 \times \tanh (2.65 z)$, and DJET $: \bar{U}=\mathrm{e}^{0.5} z \mathrm{e}^{-2 z^{2}}$, respectively.

The velocity profiles of the mean flows are shown in Fig. 1. The velocities are normalized to have the same maximum magnitude $|\bar{U}(z)|_{\max }=0.5$, and the vertical distance between $\bar{U}_{\max }$ and $\bar{U}_{\min }$ is 1 for the three mean flows. All of these $\bar{U}(z)$ are odd-symmetric about $z$ 

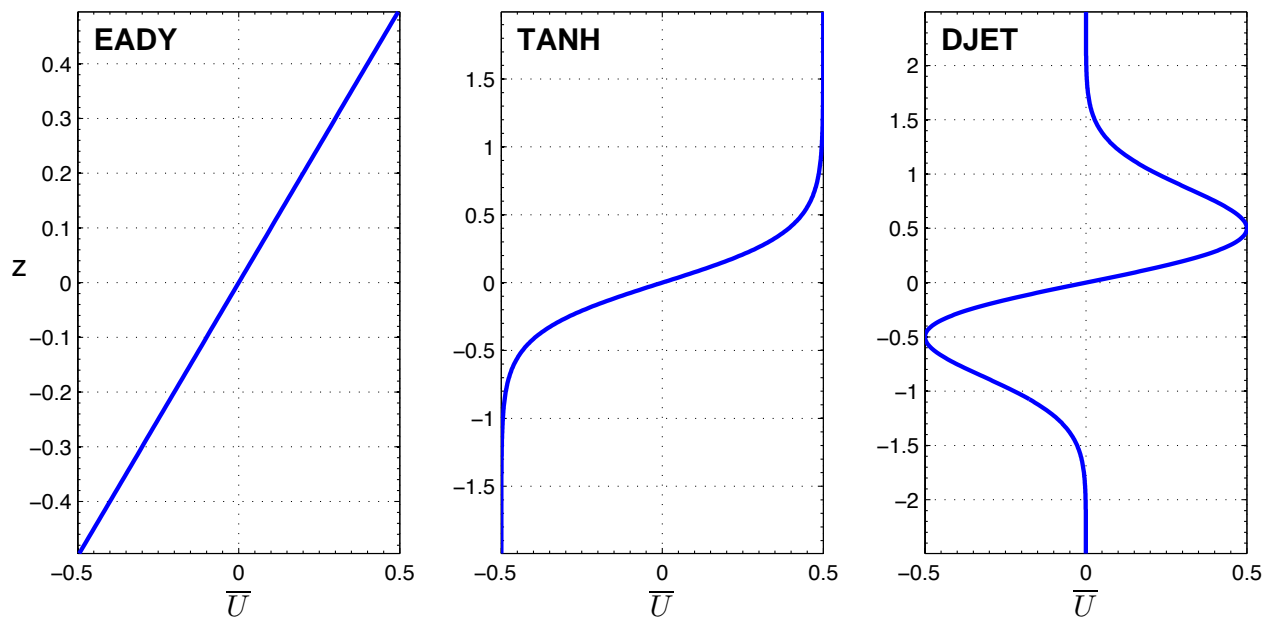

Figure 1. The mean flow velocity profiles $\bar{U}(z)$ for the EADY flow, hyperbolic tangent (TANH) flow, and the double-jet (DJET) flow. All the velocity profiles have the same maximum magnitude $(0.5)$, and the velocity difference between $[-0.5,0.5]$ is about 1 . For EADY the vertical boundaries are at $|z|=0.5$, and for the others they are at much larger $|z|$.

$=0$. With the above normalization, $U_{0}$ and $L$ represent the maximum velocity magnitude and the length scale between two extremes of the parallel shears, respectively. Because the TANH flow is simpler than the DJET flow and thus easier to understand, most our results and discussion in the next sections will be shown by this flow.

We now consider the $R o$ regimes for instabilities discussed in Sec. 1. At $R o \rightarrow 0$, the classic baroclinic instability occurs if inflection points exist (as they do here). As Ro increases, the conditions are satisfied in the following order: (i) $A-S<0$ is satisfied, and anticyclonic, ageostrophic instability (AAI) occurs or has a significant growth rate beyond this Ro; (ii) $A<0$ is satisfied, and the centrifugal instability (CI) begins to occur; (iii) the onset condition for the classic Kelvin-Helmholtz instability (KHI) (non-rotating) $R i<\frac{1}{4}$ is reached; (iv) if $N^{2}<0$, the gravitational instability would occur. The $R o$ criteria for the occurrence of AAI, CI, and KHI in these three types of mean flow are listed in Table 1 . The criteria are calculated by evaluating the $R o$ values that satisfy $\min (\bar{A}-\bar{S})=0, \min (\bar{A})=0$, and $\min (\overline{R i})=\frac{1}{4}$, respectively.

In a general form, the dimensional $A$ and $S$ in isentropic coordinates are defined as

$$
A=f+v_{X}-u_{Y} \quad \text { and } S=\sqrt{\left(u_{X}-v_{Y}\right)^{2}+\left(v_{X}+u_{Y}\right)^{2}},
$$

and their non-dimensional forms (scaled by $f$ ) are

$$
A=1+R o\left(v_{X}-u_{Y}\right) \text { and } S=R o \sqrt{\left(u_{X}-v_{Y}\right)^{2}+\left(v_{X}+u_{Y}\right)^{2}} .
$$

Here the isentropic coordinates $(X, Y, Z)$ are defined as

$$
X=x, \quad Y=y, \text { and } Z=b(x, y, z, t),
$$

and the horizontal isentropic derivatives can be written as

$$
\frac{\partial}{\partial X}=\frac{\partial}{\partial x}-\frac{b_{x}}{b_{z}} \frac{\partial}{\partial z} \text { and } \frac{\partial}{\partial Y}=\frac{\partial}{\partial y}-\frac{b_{y}}{b_{z}} \frac{\partial}{\partial z}
$$

in Cartesian coordinates $(x, y, z)$. With the coordinates transformation, the dimensional 
TABLE 1. Critical Ro for the occurrence of anticyclonic, ageostrophic instability (AAI), centrifugal instability (CI), and Kelvin-Helmholtz instability (KHI) in the three mean flows.

\begin{tabular}{llll}
\hline \hline Flow & AAI & CI & KHI \\
\hline EADY & 0.71 & 1 & 2 \\
\hline TANH & 0.53 & 0.75 & 1.51 \\
\hline DJET & 0.43 & 0.61 & 1.21 \\
\hline
\end{tabular}

$A$ and $S$ are written as

$$
A=f+v_{x}-b_{x} b_{z}^{-1} v_{z}-u_{y}+b_{y} b_{z}^{-1} u_{z}
$$

and $S=\sqrt{\left(u_{x}-b_{x} b_{z}^{-1} u_{z}-v_{y}+b_{y} b_{z}^{-1} v_{z}\right)^{2}+\left(v_{x}-b_{x} b_{z}^{-1} v_{z}+u_{y}-b_{y} b_{z}^{-1} u_{z}\right)^{2}}$

in Cartesian coordinates.

The dimensional Ertel $P V$ in Cartesian coordinates is defined as

$$
P V=\left(w_{y}-v_{z}\right) b_{x}+\left(u_{z}-w_{x}\right) b_{y}+\left(v_{x}-u_{y}+f\right) b_{z},
$$

and its non-dimensional form (scaled by $f N^{2}$ ) is

$$
P V=\frac{R o^{2}}{B u}\left(\frac{\lambda^{2} R o}{B u} w_{y}-v_{z}\right) b_{x}+\frac{R o^{2}}{B u}\left(u_{z}-\frac{\lambda^{2} R o}{B u} w_{x}\right) b_{y}+\frac{R o}{B u}\left(R_{0} v_{x}-R_{0 u}+1\right) b_{z} .
$$

When the flow is hydrostatic, we obtain that the absolute vertical vorticity in isentropic coordinates is proportional to $P V$ :

$$
P V=-v_{z} b_{x}+u_{z} b_{y}+\left(v_{x}-u_{y}+f\right) b_{z}=A b_{z} .
$$

For an interior vertical shear $\bar{U}(z)$ in our study,

$$
\bar{A}=1-R o^{2} \bar{U}_{z}^{2}, \quad \bar{S}=R o^{2} \bar{U}_{z}^{2}, \text { and } \overline{R i}=1 /\left(R o^{2} \bar{U}_{z}^{2}\right),
$$

where $A$ and $S$ are non-dimensionalized by $f$. The non-dimensional $P V$, scaled by $f N^{2}$, is

$$
\overline{P V}=1-R o^{2} \bar{U}_{z}^{2} .
$$

Note that we have dropped the term $-R o \bar{U}_{z z}$ in $\bar{B}_{z}$ with the assumption discussed earlier in the section. According to $(2.12)$ and $(2.13), \min (\bar{A}-\bar{S}), \min (\bar{A})$, and $\min (\overline{R i})$ depend on $\max \left(\bar{U}_{z}\right)$. If the mean flows were normalized to have the same maximum mean shear $\max \left(\bar{U}_{z}\right)$, the $R o$ criteria in Table 1 would be the same for all of these three. The normalization that we choose keeps the mean flows with the same bulk $\Delta U / \Delta H$, but not the local values, hence the $R o$ criteria are different for them. The normalization of the mean flows is an arbitrary but free choice.

\section{Diagnosis of the unstable modes}

In the following sections, we show the main characteristics of two types of ageostrophic instability in the vertical shear flows. Besides the growth rate, frequency, Ro dependence, 
and eigenmode patterns, we also diagnose the energetics and the momentum balance of the unstable modes. This section briefly introduces how the diagnosis calculations are performed.

\subsection{Energetics}

In the Boussinesq equations, the non-dimensional kinetic energy for the perturbation is defined by $\mathrm{KE}=\frac{1}{2} \int\left(\overline{u^{\prime 2}}+\overline{v^{\prime 2}}+\lambda^{2} R o^{2} \overline{w^{\prime 2}}\right) d z$, and the available potential energy is $\mathrm{APE}=\frac{1}{2} \int \overline{b^{2}} d z$. From (2.1), we derive the perturbation energy balance equations with a mean flow $\bar{U}(z)$ :

$$
\left\{\begin{array}{c}
\frac{d \mathrm{KE}}{d t}=\int\left[-R_{o} \bar{U}_{z} \overline{u^{\prime} w^{\prime}}+\overline{w^{\prime} b^{\prime}}\right] d z \\
\frac{d \mathrm{APE}}{d t}=\int\left[\overline{U_{z}} \overline{v^{\prime} b^{\prime}}-\overline{w^{\prime} b^{\prime}}\right] d z
\end{array}\right.
$$

The term $\int-R o \bar{U}_{z} \overline{u^{\prime} w^{\prime}} d z$ is the vertical Reynolds stress work, indicating the vertical energy conversion from the mean kinetic energy to the perturbation kinetic energy (KMKE); the term $\int \bar{U}_{z} \overline{v^{\prime} b^{\prime}} d z$ is the horizontal buoyancy flux work, indicating the energy transfer from the mean potential energy to the perturbation potential energy (PMPE). $\int \overline{w^{\prime} b^{\prime}} d z$ represents the energy conversion between the potential and kinetic energy in the perturbation (PEKE); a positive value represents an energy transfer from potential to kinetic. The mean eddy energy conversion demonstrates a typical characteristic of an instability, which could show the difference among the unstable modes we examine. For instance, the classic BCI grows by drawing the potential energy from the mean state, while the AI1 ageostrophic instabilities in Sec. 4 draws kinetic energy from the mean state, which clearly differ from the BCI.

\subsection{Diagnosis of balance}

We analyze the degree of balance by the method devised in Molemaker et al. (2005) (see Section 5 and Appendix $\mathrm{C}$ in their paper). First, we derive the higher-order $\mathrm{O}\left(R o^{2}\right)$ Balanced Equations in Cartesian coordinates. We define the streamfunction $\psi$ and the velocity potential $\chi$ as

$$
u=-\frac{\partial \psi}{\partial y}+R o \frac{\partial \chi}{\partial x} \text { and } v=\frac{\partial \psi}{\partial x}+R o \frac{\partial \chi}{\partial y},
$$

decompose them into the mean and the perturbation components,

$$
\psi=\bar{\Psi}+\psi^{\prime}, \quad \chi=\bar{X}+\chi^{\prime},
$$

and substitute the perturbation component of (3.2) into (2.1). By taking the curl and divergence of the horizontal momentum equations, respectively, we replace the first two equations in (2.1) by the vorticity and divergence equations:

$$
\left\{\begin{aligned}
\left(\frac{\partial}{\partial t}+\bar{U} \frac{\partial}{\partial x}\right) \nabla_{h}^{2} \psi^{\prime}-R o \bar{U}_{z} \frac{\partial w^{\prime}}{\partial y}+\nabla_{h}^{2} \chi^{\prime} & =0 \\
R o^{2}\left(\frac{\partial}{\partial t}+\bar{U} \frac{\partial}{\partial x}\right) \nabla_{h}^{2} \chi^{\prime}+R o^{2} \bar{U}_{z} \frac{\partial w^{\prime}}{\partial x}-\nabla_{h}^{2} \psi^{\prime}+\nabla_{h}^{2} p^{\prime}+R o^{3} \nabla_{h}^{4} \chi^{\prime}= & 0 .
\end{aligned}\right.
$$

Dropping all the terms with order $R o^{2}$ or higher, we obtain the linearized higher-order Balanced Equations analogous to that in isentropic coordinates in McWilliams et al. 
(1998):

$$
\left\{\begin{aligned}
\left(\frac{\partial}{\partial t}+\bar{U} \frac{\partial}{\partial x}\right) \nabla_{h}^{2} \psi_{b a l}^{\prime}-R o \bar{U}_{z} \frac{\partial w_{b a l}^{\prime}}{\partial y}+\nabla_{h}^{2} \chi_{b a l}^{\prime} & =0 \\
\nabla_{h}^{2} \psi_{b a l}^{\prime} & =\nabla_{h}^{2} p_{b a l}^{\prime} \\
b_{b a l}^{\prime} & =\frac{\partial p_{b a l}^{\prime}}{\partial z} \\
\nabla_{h}^{2} \chi_{b a l}^{\prime}+\frac{\partial w_{b a l}^{\prime}}{\partial z} & =0 \\
\left(\frac{\partial}{\partial t}+\bar{U} \frac{\partial}{\partial x}\right) b_{b a l}^{\prime}+\bar{B}_{y} v_{b a l}^{\prime}+w_{b a l}^{\prime} & =0
\end{aligned}\right.
$$

where $\nabla_{h}^{2}$ denotes the horizontal Laplace operator. We decomposed the perturbation variables into balanced and unbalanced components, $X^{\prime}=X_{b a l}^{\prime}+X_{u n b}^{\prime}$, and assume $X^{\prime}$ $\left(\psi^{\prime}, \chi^{\prime}, w^{\prime}, p^{\prime}\right.$, or $\left.b^{\prime}\right)$ has a $\operatorname{Re}\left[\hat{X}(z) e^{i(k x+l y)} e^{\tilde{\sigma} t}\right]$ form. Eliminating the time derivatives by combining the vorticity and buoyancy equations in (3.4), we finally get the diagnostic constraints of the Balanced Equations:

$$
\left\{\begin{aligned}
2 i k \bar{U}_{z} \hat{\psi}_{b a l}+\left(2 i l R o \bar{U}_{z}+\frac{\partial}{\partial z}\right) \hat{\chi}_{b a l}+\left(\frac{i l R o \bar{U}_{z z}}{k^{2}+l^{2}}-1\right) \hat{w}_{b a l} & =0 \\
\hat{\psi}_{b a l} & =\hat{p}_{b a l} \\
\hat{b}_{b a l} & =\frac{\partial \hat{p}_{b a l}}{\partial z} \\
\left(k^{2}+l^{2}\right) \hat{\chi}_{b a l} & =\frac{\partial \hat{w}_{b a l}}{\partial z}
\end{aligned}\right.
$$

Following the same way as Molemaker et al. (2005) (Appendix C), the full perturbation solution is projected onto the constraints of Balanced Equations (3.5) to maximize the balanced component. Here energy $\hat{E}=\int \frac{1}{2}\left(|\hat{u}|^{2}+|\hat{v}|^{2}+\lambda^{2} R o^{2}|\hat{w}|^{2}+|\hat{b}|^{2}\right) d z$ is chosen as a metric for unbalance, and the minimized degree of unbalance $\delta$ is defined by the integrated energy ratio,

$$
\delta=\frac{\frac{1}{2} \int\left[\left|\hat{u}_{\mathrm{unb}}\right|^{2}+\left|\hat{v}_{\mathrm{unb}}\right|^{2}+\lambda^{2} R o^{2}\left|\hat{w}_{\mathrm{unb}}\right|^{2}+\left|\hat{b}_{\mathrm{unb}}\right|^{2}\right] d z}{\frac{1}{2} \int\left[|\hat{u}|^{2}+|\hat{v}|^{2}+\lambda^{2} R o^{2}|\hat{w}|^{2}+|\hat{b}|^{2}\right] d z}
$$

where $|\hat{X}|$ denotes the absolute value of $\hat{X}$.

Note that to diagnose $\hat{X}_{b a l}$, we actually project the full eigenfunction $\hat{X}$ that satisfies (2.5) onto the balanced constraints (3.5) after eliminating the time derivatives in (3.4), rather than directly solving the following balanced eigenvalue problem:

$$
\left\{\begin{aligned}
\left(i k \bar{U}+\tilde{\sigma}_{b a l}\right)\left(k^{2}+l^{2}\right) \hat{\psi}_{b a l}+\left(k^{2}+l^{2}\right) \hat{\chi}_{b a l}+i l R o \bar{U}_{z} \hat{w}_{b a l} & =0 \\
\hat{\psi}_{b a l} & =\hat{p}_{b a l} \\
\hat{b}_{b a l} & =\frac{\partial \hat{p}_{b a l}}{\partial z} \\
\left(k^{2}+l^{2}\right) \hat{\chi}_{b a l} & =\frac{\partial \hat{w}_{b a l}}{\partial z} \\
\left(i k \bar{U}+\tilde{\sigma}_{b a l}\right) \hat{b}_{b a l}-i k \bar{U}_{z} \hat{\psi}_{b a l}-i l R o \bar{U}_{z} \hat{\chi}_{b a l}+\hat{w}_{b a l} & =0,
\end{aligned}\right.
$$

This does not guarantee that the diagnosed $\hat{X}_{b a l}$ is consistent with a balanced normal mode solution of (3.4) with a form $X_{b a l}^{\prime}=\operatorname{Re}\left[\hat{X}(z) e^{i(k x+l y)} e^{\tilde{\sigma}_{b a l} t}\right]$, i.e., the diagnosed $\hat{X}_{b a l}$ that satisfies (3.5)-(3.6) is not necessarily an eigenmode solution of the Balanced 
Equations (3.7). However if $\hat{X}$ has an unbalanced component with the above projection, it is still true that $X^{\prime}$ is not a solution of (3.4). We test this argument by substituting the diagnosed $\hat{X}_{b a l}$ into (3.7) to diagnose the value of $\tilde{\sigma}_{b a l}$. If $\hat{X}$ is higher-order balanced, then the diagnosed $\tilde{\sigma}_{b a l}(z)$ is a constant value that equals $\tilde{\sigma}$, and the diagnosed $\hat{X}_{b a l}$ is the eigenmode solution to both (2.5) and (3.7). We have verified this for a BCI mode at small Ro. Otherwise, if $\hat{X}$ has an unbalanced component $(\delta>0)$, there is no common $\hat{X}_{b a l}$ satisfies both (3.5) and (3.7) for a constant $\tilde{\sigma}_{b a l}$ even when $\delta$ is small but nonzero; hence $\hat{X}$ is not an approximate eigenmode solution to the higher-order Balanced Equations. Therefore, the unstable mode with $\delta \neq 0$ is unbalanced, and the magnitude of $\delta$ represents the degree of unbalance.

\section{AI1: Continuous ageostrophic transformation between BCI and CI}

For the three types of $\bar{U}(z)$ with a zero vertical average (Fig. 1), the dominant unstable mode has a vertically symmetric structure, with zero frequency (the eigenvalue $\tilde{\sigma}$ of this mode only has a real part, with $\omega=0)$, and the eigenfunction satisfies $\{\hat{u}, \hat{v}, \hat{w}, \hat{p}, \hat{b}\}(z)=$ $i\left\{\hat{u}^{*}, \hat{v}^{*}, \hat{w}^{*},-\hat{p}^{*}, \hat{b}^{*}\right\}(-z)$, where ${ }^{*}$ denotes complex conjugate. In the quasigeostrophic regime, this mode is the classic baroclinic instability (BCI) mode. As Ro increases, this vertically symmetric mode becomes less unstable near $l=0$ in the EADY flow, and its occurrence shrinks to smaller $k$, while in the TANH and DJET flows, it becomes slightly more unstable near $l=0$ and extends to larger $k$. On the other hand, with increasing $R o$, these vertically symmetric modes extend to larger $l$ and continuously change their characteristics in all three mean flows.

\subsection{AI1 in TANH flow}

To be representative of the continuous transformation, the growth rate of the vertically symmetric mode $(\omega=0)$ in the TANH flow in a $(k, l)$ space with increasing Ro is shown in Fig. 2. Note that this vertically symmetric mode occurs in a single $(k, l)$-connected region with continuous transformation of its properties as $(k, l, R o)$ vary, rather than multiple, coexisting but disconnected modes with the same frequency $\omega=0$. (The result is confirmed by a full-spectrum eigenvalue solver with a lower resolution.) The maximum growth rate for each $R o$ is denoted by a cross symbol in the subplot, and the lower limit of $l$ (calculated from (2.6)) for each $R o$ above which the perturbations are safely calculated with the "wide jet approximation" is shown by the white horizontal line. The integrated energy conversion terms PMPE and KMKE for the same $(k, l)$ space and $R o$ are shown in Fig. 3 and 4, respectively. In the $R o \rightarrow 0$ limit $(R o=0.01)$, only the BCI mode exists, and its growth rate is the strongest near $l=0$. For small $R o$, the mean potential energy conversion (PMPE) is the dominant energy source for the BCI perturbation to grow. The growth rate of the BCI mode slightly increases with increasing Ro. The contribution of the mean kinetic energy conversion (KMKE) increases, but it is still much less than the PMPE conversion for the BCI mode.

As $R o$ increases, the appearance of this vertically symmetric mode broadens to larger $k$ and $l$ and its properties change substantially. Away from the region near $l=0$, the mode has a positive KMKE conversion and a negative PMPE conversion, indicating that the main energy source for the perturbation is mean kinetic energy, and kinetic energy is converted to potential energy by the perturbation. This mode is clearly distinguished from the BCI mode by its energetics, and it starts to occur at a finite $R o$ that is far below the criterion for CI. It is a newly identified type of ageostrophic instability; we name it AI1. At $R o=0.7$ and 0.75 , the AI1 mode has extended to very large $l(>15)$ 

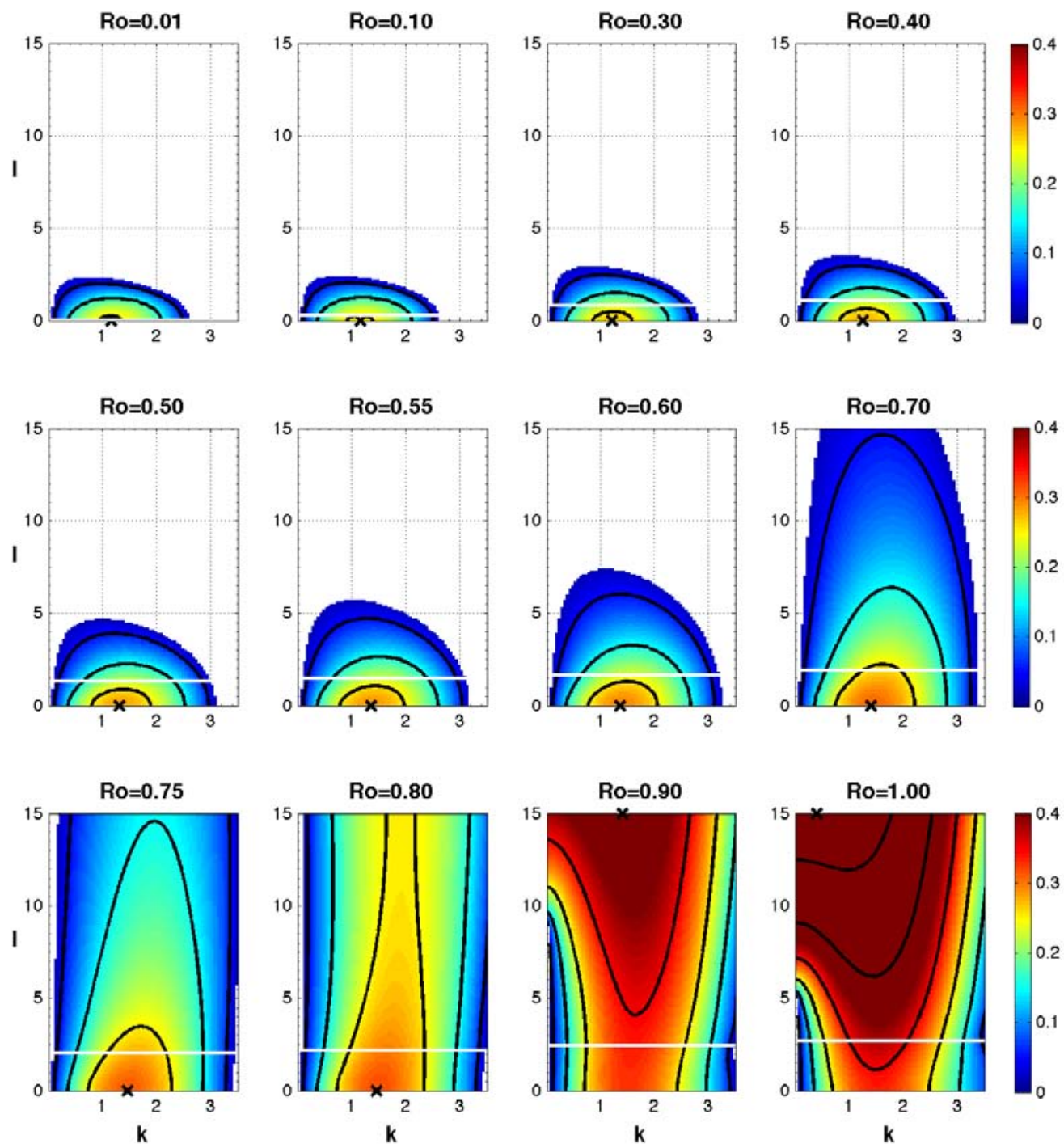

FIGURE 2 . The growth rate of the vertically symmetric mode $(\omega=0)$ in the TANH flow in a $(k$, $l$ ) space with increasing $R o$. The minimum value for the contours is 0.05 , and their interval is 0.1 . The $(k, l)$ for the maximum growth rates for each $R o$ are denoted by the cross symbols in the subplots. As $R o$ increases, the values for $\sigma_{\max }$ are $0.26,0.26,0.27,0.27,0.28,0.29,0.29,0.31$, $0.31,0.32,0.44,0.60$, respectively. The white horizontal line in each $R o$ subplot denotes the lower limit of $l$ above which the perturbations are safely calculated with the "wide jet approximation". $A-S=0$ at $R o=0.53$ and $A(P V)=0$ at $R o=0.75$.

and become highly ageostrophic (with large horizontal divergence and vertical velocity, Fig. 8) at intermediate $k$ and large $l$.

When the $R o$ is above the criterion for CI $(R o>0.75, P V<0)$, the CI mode begins to appear near $k=0$. For a fixed $R o$, there is a lower limit in $l$ for the occurrence of CI, 

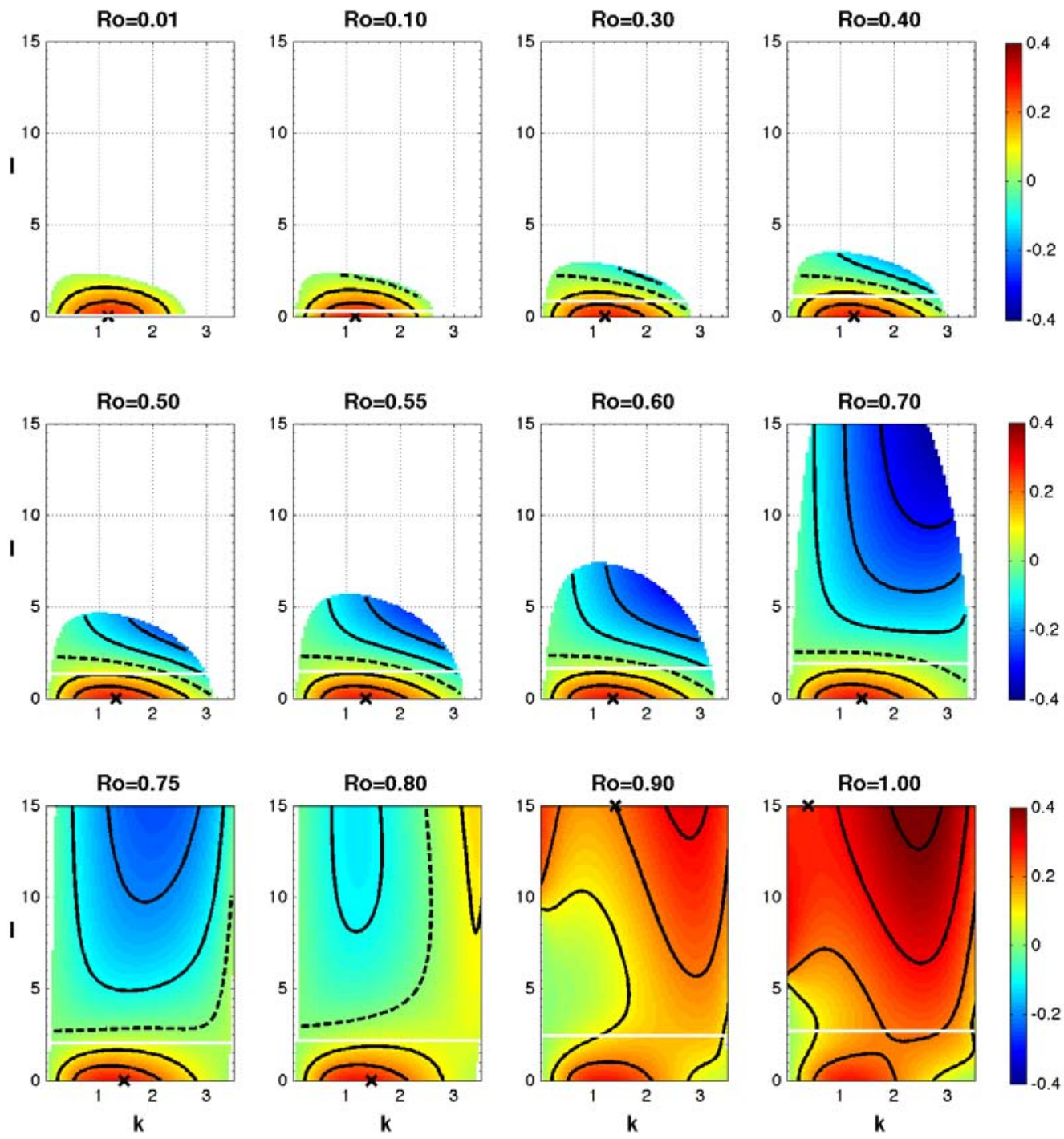

FIGURE 3. The integrated energy conversion from mean potential to perturbation potential (PMPE) of the vertically symmetric mode $(\omega=0)$ in the TANH flow in a $(k, l)$ space with increasing Ro. The interval of the contours is 0.1 and the zero value contour is shown by the dash line. The $(k, l)$ for the maximum growth rates for each $R o$ are denoted by the cross symbols in the subplots. The white horizontal line in each $R o$ subplot denotes the lower limit of $l$ above which the perturbations are safely calculated with the "wide jet approximation". $A-S=0$ at $R o=0.53$ and $A(P V)=0$ at $R o=0.75$.

and the growth rate increases with $l$. At $R o=0.8$, the CI mode appears approximately at $l>25$ and hence is not shown in the Fig. 2. The lower limit in $l$ for the CI mode decreases with increasing $R o$, as shown in the $R o=0.9$ and 1 subplots. Consistent with the Stone (1966) result, the unstable CI mode extends to $l \rightarrow \infty$ in the non-viscous case. 

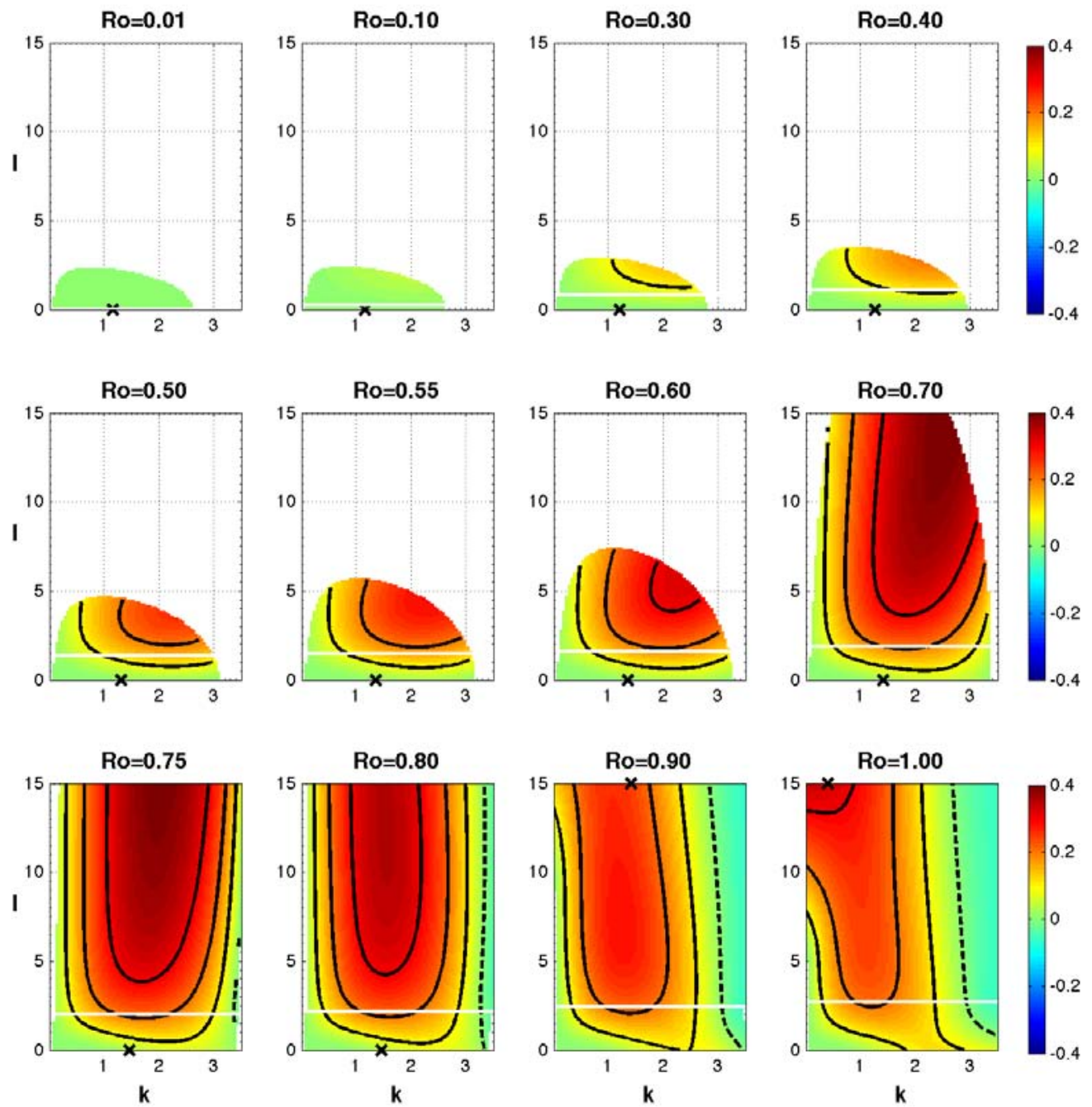

FigURE 4. The integrated energy conversion from mean kinetic to perturbation kinetic (KMKE) of the vertically symmetric mode $(\omega=0)$ in the TANH flow in a $(k, l)$ space with increasing Ro. The interval of the contours is 0.1 and the zero value contour is shown by the dash line. The $(k, l)$ for the maximum growth rates for each $R o$ are denoted by the cross symbols in the subplots. The white horizontal line in each $R o$ subplot denotes the lower limit of $l$ above which the perturbations are safely calculated with the "wide jet approximation". $A-S=0$ at $R o=$ 0.53 and $A(P V)=0$ at $R o=0.75$.

The PMPE and KMKE are both positive for a CI mode, indicating the perturbations draw energy from both the mean potential and kinetic energy. The main energy source is the mean potential energy conversion at relatively small $R o$ or $l$. As $R o$ or $l$ increases, the mean kinetic energy conversion becomes dominant. Similar conclusions on the energetics of a CI mode can be deduced from the analytical solution in Hoskins (1974), for the symmetric instability of a uniform vertical shear flow. 


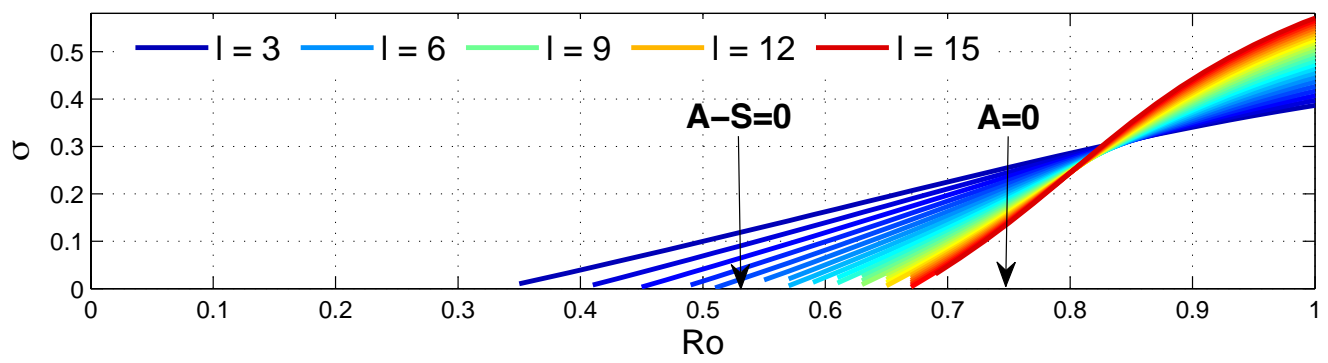

FIgURE 5. The growth rate $\sigma$ of the vertically symmetric mode as a function of $R o$ for $k=1.5$ and $l=3-15$ (interval $=0.5$ ) in the TANH flow. Below around $R o=0.8$, the mode is a AI mode for $l \geqslant 3$ with a negative PMPE conversion. When $R o>0.8$, the mode transforms to a CI mode.

Beginning from around $R o=0.8$, another type of ageostrophic instability arises at intermediate $k(k>3)$. This mode has a positive mean potential energy conversion and a negative mean kinetic energy conversion, which differs from BCI, AI1, and CI. Its mode structure is associated with inertia critical layers, hence is a type of AI2, which will be discussed in Sec. 5.

At $R o=0.9$ and 1 , all the unstable modes (the above four types) coexist, occuring over small and intermediate $k(k<4)$, and a wide range of $l$. Because the growth rate of CI strongly increases with $R o$ and its appearance extends to non-zero $k$, it dominates over AI1 at intermediate and large $l(l>5)$ for $R o \geqslant 0.9$, with the maximum growth rate occurring at small non-zero $k$ and large $l(l>15$, not shown in Fig. 2). The PMPE conversion becomes positive as the CI dominates in those $(k, l)$ regions.

The AI1 mode appears in a continuous transformation of unstable mode properties between BCI and CI. It does not exist in the quasigeostrophic equations, or even higherorder Balanced Equations (Fig. 6). It is an ageostrophic instability that occurs at finite $R o$, but below the onset for CI, and its growth rate increases with increasing $R o$. Though it does not clearly separate from the BCI and CI in $(k, l)$, the energetics of the three types of instability are distinguishable for an intermediate Ro (Fig. 3 and 4). For $R o<0.75$, the potential energy conversion is positive near $l=0$ (where BCI is the strongest); the negative potential energy conversion region roughly encloses the $(k, l)$ regime for AI1. At $R o=0.8$, the potential energy starts to become positive near $k=0$ for large $l$ (not shown in Fig. 3) as CI starts to occur. For even larger Ro values, the CI extends to smaller- $l$ and larger- $k$ directions; and the potential energy conversion becomes positive where CI is dominant over AI1. The potential energy conversion is positive at intermediate $k$ ( $k$ $>3$ ) for $R o \geqslant 0.8$ due to the occurrence of AI2. Because AI1 is not clearly separated with BCI or CI, the onset $R o$ for the AI1 mode is not sharply determined. In Fig. 2, the growth rate of AI1 and its occurrence in $(k, l)$ has a strong increase nearby $R o=$ $0.53(A-S=0)$ but below $R o=0.75(A=0)$, which allows its categorization as a type of AAI. Fig. 5 shows the growth rate of the vertically symmetric mode as a function of Ro. The growth rate of AI1 is smaller than BCI or CI, with the strongest unstable AI1 mode occurring far from $l=0$ or $k=0$. We choose $k=1.5$ and $l=3-15$, in which regime AI1 is dominant and with a negative PMPE conversion below $R o=0.8$. The AI1 mode starts to appear in the vicinity of $A-S=0$ and well below $A=0$ in Fig. 5, which confirms its possible categorization as AAI. The mode transforms to a CI mode in this $(k, l)$ region when $R o>0.8$, and the PMPE conversion becomes positive, as shown in Fig. 2 and 3.

The degree of unbalance $\delta$ (defined in Sec. 3.2) of this vertically symmetric mode 

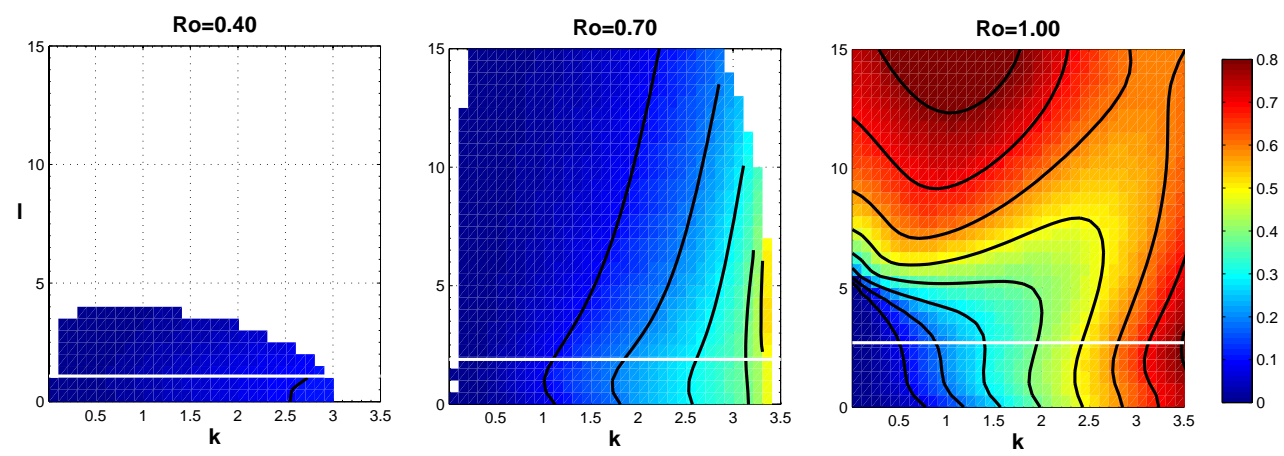

Figure 6 . The degree of unbalance $\delta$ on a $(k, l)$ space for $R o=0.40,0.70$, and 1.00 , respectively. The minimum value for the contours is 0.1 , and their interval is 0.1 . Only regions with $\sigma>0$ are contoured. The white horizontal line in each $R o$ subplot denotes the lower limit of $l$ above which the perturbations are safely calculated with the "wide jet approximation".

in TANH flow for $R o=0.4,0.7$ and 1 in $(k, l)$ space is shown in Fig. 6. For $R o<$ 0.4 , the mode is mostly a BCI mode and it is mostly balanced. As Ro increases, AI1 begins to occur, and the mode becomes unbalanced at intermediate $k$. At $R o=0.7$, the BCI mode (for small $k$ near $l=0$ ) is mostly balanced $(\delta \sim 0.1)$. The AI1 mode has a very small unbalanced component for small $k$ even at large $l$; the unbalanced fraction increases as it extends to larger $k$. Due to the existence of unbalance at the beginning of its occurrence, the AI1 mode does not exist in the higher-order Balanced Equations (3.7) (discussed in the last paragraph of Sec. 3.2). This fact, together with other properties of AI1 (e.g., negative PMPE and positive KMKE), indicates that as the mode continuously transforms, it changes to another mode with different characteristics rather than simply an "ageostrophic" BCI mode. At Ro =1, the CI mode occurs at intermediate and large $l$; it is highly unbalanced. The AI2 mode $(k>3)$ is also highly unbalanced, due to its association with inertia-gravity waves (Sec. 5).

The eigenmode patterns for typical BCI, AI1 and CI modes in TANH flow are shown in Fig. 7 to 9, respectively. The perturbation variables are normalized by the square root of the vertically averaged energy:

$$
E^{\prime}=\frac{1}{H_{0}} \int \frac{1}{2}\left(\overline{u^{\prime 2}}+\overline{v^{\prime 2}}+\lambda^{2} R o^{2} \overline{w^{\prime 2}}+\overline{b^{\prime 2}}\right) d z
$$

i.e., all the variables are divided by $\sqrt{E^{\prime}}$, and the normalized variables, including their full, balanced, and unbalanced components, are plotted with the same color scale. Here $H_{0}$ represents the vertical domain size. Because the mode patterns of the vertically symmetric mode transforms continuously in $(k, l)$ and $R o$, we here choose to show the mode pattern of each mode in its typical $(k, l, R o)$ regime discussed above. For a BCI mode (Fig. 7) close to $l=0, u^{\prime}$ has a small magnitude due to the geostrophic balance. The perturbation $v^{\prime}$ is mostly in phase with $b^{\prime}$, while the phase correlation between $u^{\prime}$ and $w^{\prime}$ is weak, which shows a dominance of PMPE. As in a quasigeostrophic solution, the streamfunction (not shown in Fig. 7, but can be inferred from $u^{\prime}$ and $v^{\prime}$ ) shows an upstream (westward) phase tilt with height to allow $w^{\prime} b^{\prime}>0$ (Pedlosky 1979). The BCI mode is nearly balanced for $R o=0.1$, and even for $R o=0.5$ ( $\delta$ is around 0.08, not shown in Fig. 7). Opposite to BCI, an AI1 mode (Fig. 8) has a downstream phase tilt with height. There is a $180^{\circ}$ phase shift between $w^{\prime}$ and $b^{\prime} ; u^{\prime}$ and $w^{\prime}$ are negatively correlated, and $v^{\prime}$ and $b^{\prime}$ are negatively correlated, implying a negative PMPE and a positive KMKE. The AI1 mode has a small but finite unbalanced fraction in every variable. The CI mode (Fig. 9) is highly 

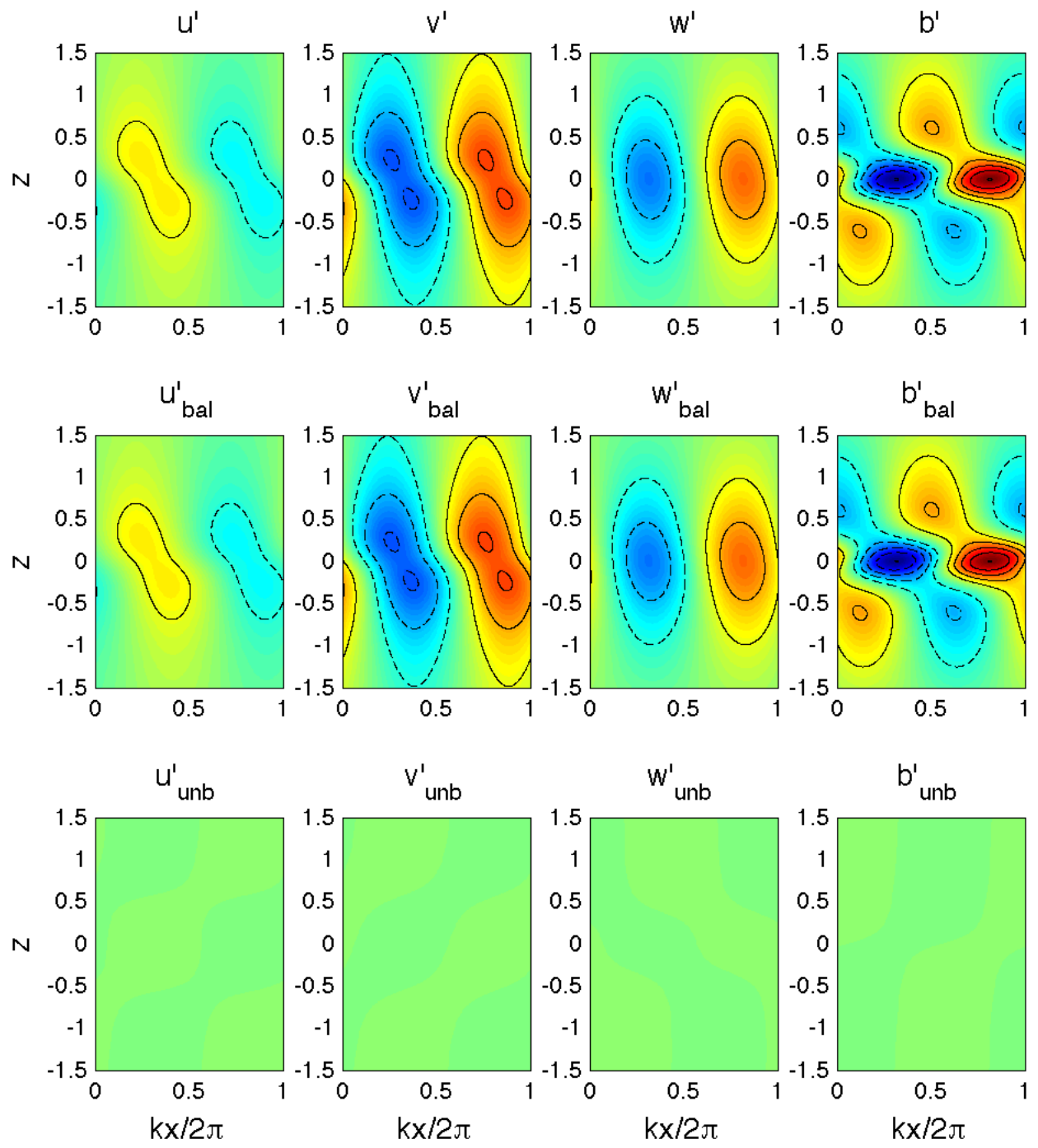

FigURE 7. Normalized perturbation patterns $\left(u^{\prime}, v^{\prime}, w^{\prime}, b^{\prime}\right)$ of a classic baroclinic instability (BCI) mode (vertically symmetric) in the TANH flow. The total perturbation variables, balanced components, and unbalanced components are shown in the upper, middle and lower rows, respectively. All the subplots are with the same color scale. Red (solid contours) represents positive, and blue (dashed contours) represents negative. The shown BCI mode is for $k=1.2, l=$ 0.5 , and $R o=0.1$; its unbalanced fraction $\delta=0.0025$ and growth rate $\sigma=0.24$.

unbalanced especially in $v^{\prime}$ and $w^{\prime}$, which implies a strong inertial momentum balance in the zonal direction. The phase correlation between $u^{\prime}$ and $w^{\prime}$, or $v^{\prime}$ and $b^{\prime}$, necessary for positive KMKE, or PMPE, is not easily identified in the eigenmode patterns, which have both in-phase and out-of-phase components. 

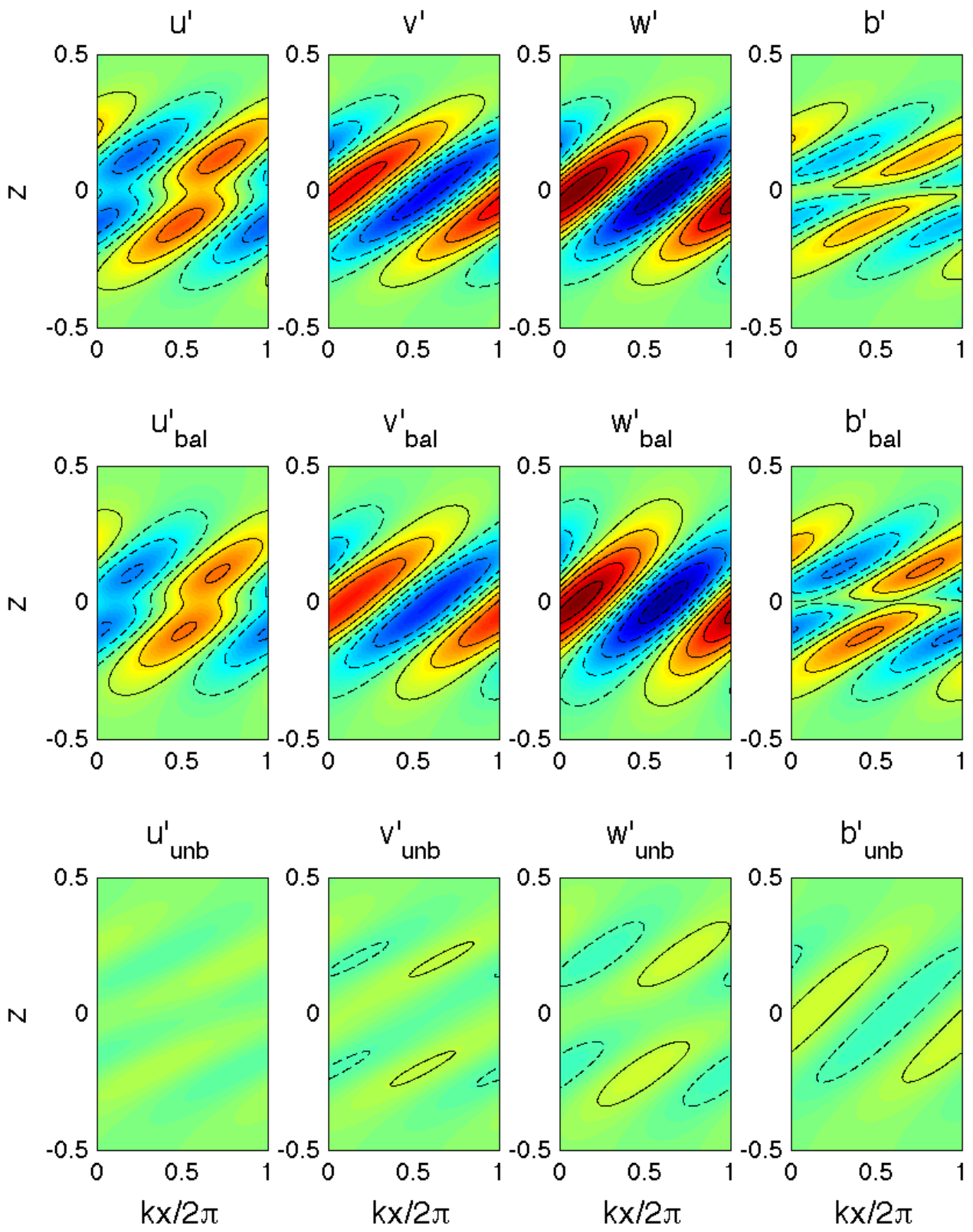

FiguRE 8. Normalized perturbation patterns $\left(u^{\prime}, v^{\prime}, w^{\prime}, b^{\prime}\right)$ of a first type of ageostrophic instability (AI1) mode (vertically symmetric) in the TANH flow. The plot conventions are the same as in Fig. 7. The shown AI1 mode is for $k=2.5, l=12$, and $R o=0.75$; its unbalanced fraction $\delta=0.23$ and growth rate $\sigma=0.15$. 

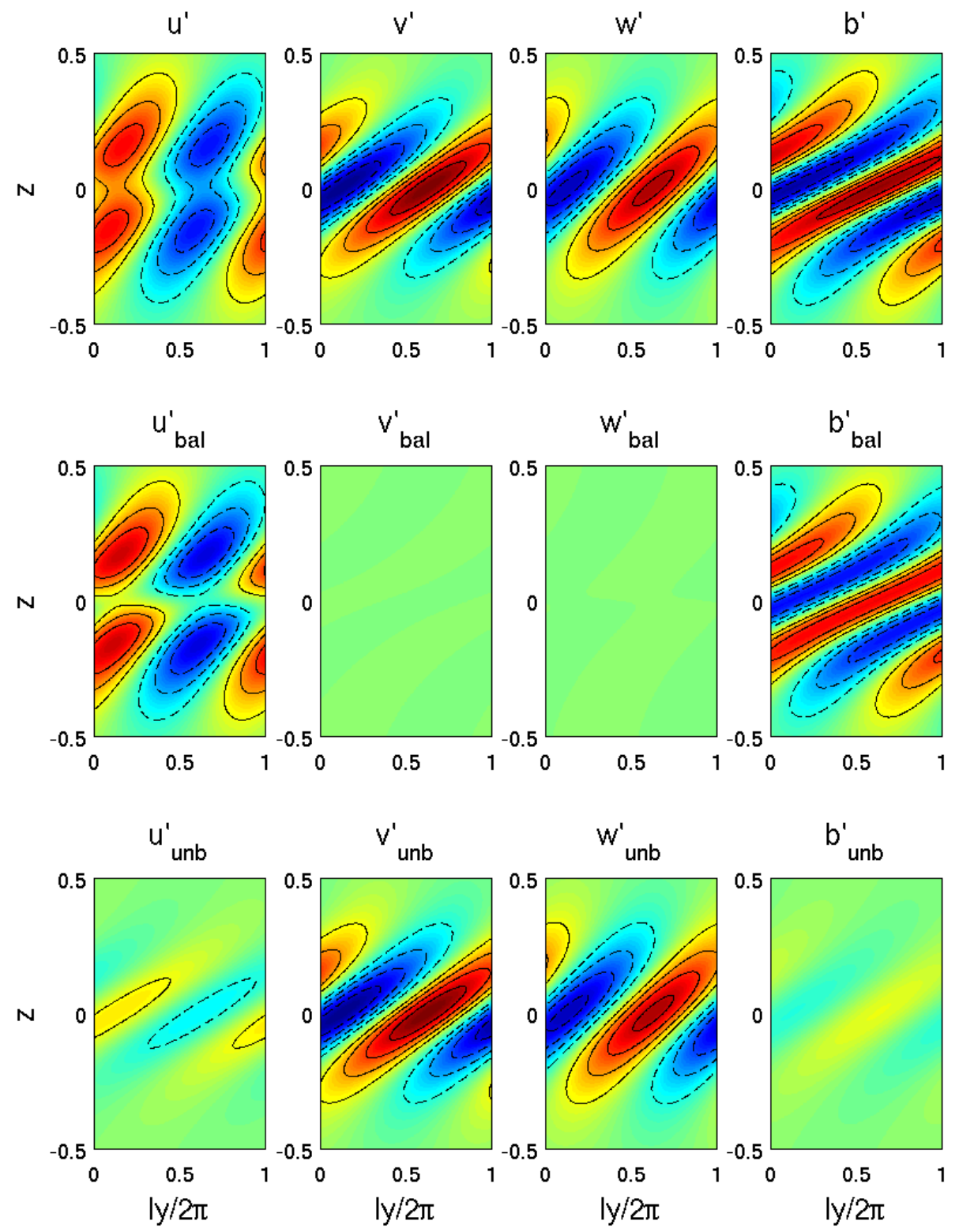

FiguRE 9. Normalized perturbation patterns $\left(u^{\prime}, v^{\prime}, w^{\prime}, b^{\prime}\right)$ of a centrifugal instability (CI) mode (vertically symmetric) in the TANH flow. The plot conventions are the same as in Fig. 7. The shown CI mode is for $k=0, l=9$, and $R o=1.0$; its unbalanced fraction $\delta=0.60$ and growth rate $\sigma=0.45$. 


\subsection{AI1 in other flows}

The behavior of this vertically symmetric mode in the EADY and DJET flows is similar to that in the TANH flow, except that in the EADY flow, all the modes appear in a much narrower band of small $k$, especially when $l$ is large. The growth rate of the mode in a $(k, l)$ space for the three types of flows with increasing $R o$ is shown in Fig. 10. In the first three columns, the AI1 clearly appears in the intermediate and large $l$ region, and it has a positive KMKE conversion and a negative PMPE conversion for all these three flows (energy conversions not shown here). As Ro increases in Fig. 10, $P V$ changes from positive to negative; the $R o$ in the second column are the onset criteria for CI, which occurs at infinite large $l$ with vanishing viscosity at $P V=0$. The AI1 is robust as $P V>0$ (the first column), and it continues to be dominant in the shown $(k, l)$ region until $P V$ is slightly negative (the second and third columns). As $P V$ is strongly negative (the fourth column), the CI becomes the dominant mode in this $(k, l)$ region. In sum, as in the TANH flow, the AI1 mode in the EADY and DJET flows also appears in a continuous transformation between the BCI and CI modes. The white horizontal line in each subplot denotes the lower limit of $l$ above which the perturbations are safely calculated with the "wide jet approximation". For the EADY flow, $\overline{U_{z z}}$ is always zero, so the results are all accurate solutions to (2.1).

As stated in Sec. 1, the EADY flow has been examined by Stone $(1966,1970)$ and Molemaker et al. (2005). We have reproduced their results to compare the AI1 mode (not shown here). Both of them have seen the AI1 mode in the examination of the strongest unstable mode with finite $R o$ and intermediate cross-stream wavenumbers, e.g., Fig. 2 and Fig. $3(\lambda \geqslant 4)$ in Stone (1970), and Fig. $1(k \geqslant 4)$ in Molemaker et al. (2005), but they did not conduct a full search on the $(k, l)$ space of this vertically symmetric mode, and did not check the mode properties (e.g., energy conversion) of it; hence the continuous transformation of modes and the AI1 mode was not identified. Note that the non-dimesionalization in Stone $(1966,1970)$ is different from ours, the detailed parameter comparison between his and our results is in Appdendix A. Our focus is the ageostrophic instability occurring for not too large $R o$ (below the criterion of KHI), so we do not explore mean flows with $R i<\frac{1}{4}$, which Stone $(1966,1970)$ did.

Because we study flows with a very small $\lambda=0.01$, all the unstable modes discussed above are mostly hydrostatic (not shown). We have checked the above numerical results for TANH and DJET flows upon the validity of the "wide jet approximation" made in Sec. 2. As shown in Fig. 2 to 10, the perturbations with $l$ above the white lines are reliable with this approximation, which means all the ageostrophic modes of interest, AI1, CI, and intermediate and large $l$ AI2 modes are safely calculated. Due to the failure of "wide jet" approximation, the BCI mode is not reliably calculated near $l=0$ for large $R o$, but this does not affect our main conclusions on the ageostrophic instabilities.

\section{AI2: Ageostrophic instability associated with ICL}

Besides the zero-frequency unstable modes discussed in Sec. 4, we find other ageostrophic modes in the TANH and DJET flows, which all appear with inertia critical layers (ICL). The inertia critical layer is defined as where $k \bar{U}(z)-\omega= \pm f$ dimensionally, or $k \bar{U}(z)-\omega= \pm 1 / R o$ non-dimensionally. The physical characteristics of ICL have been recognized as an "absorber" of inertia-gravity waves (Jones 1967), and Yamanaka (1985) shows that inertia-gravity waves in an ICL can be unstable due to a non-uniform mean flow. We categorize these modes as another type of ageostrophic instability: AI2. AI2 has a weaker growth rate than AI; and with $\lambda=0.01$, AI2 is also mostly hydrostatic. 

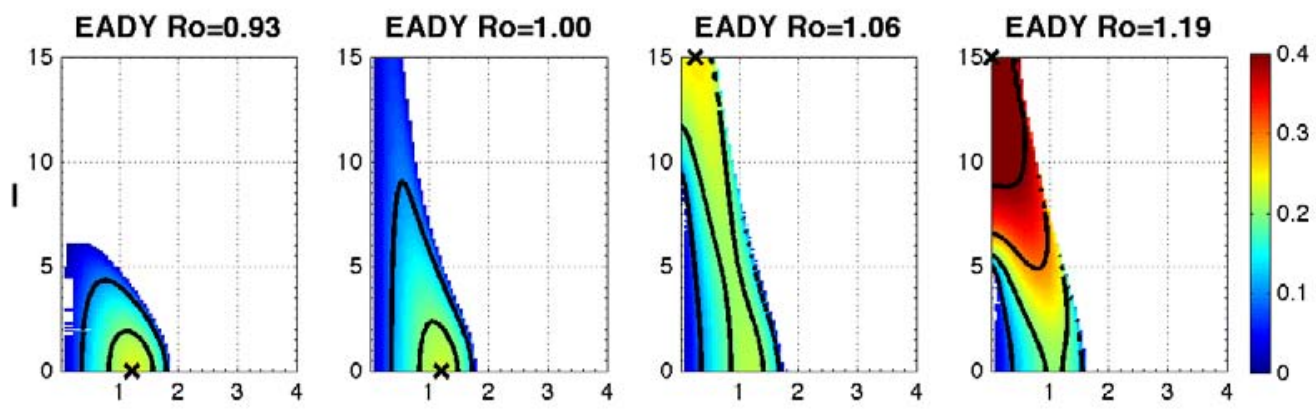

TANH Ro $=0.70$

TANH Ro $=0.75$
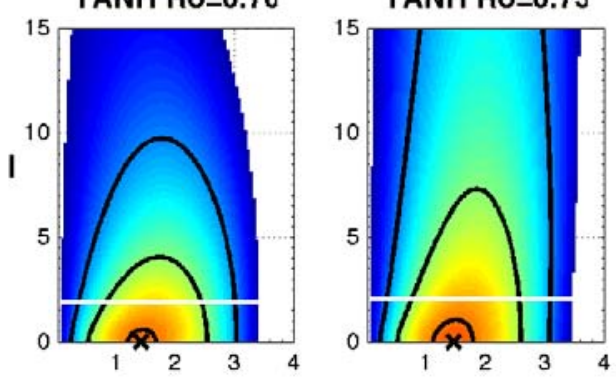

TANH Ro $=0.80$

TANH Ro $=0.90$
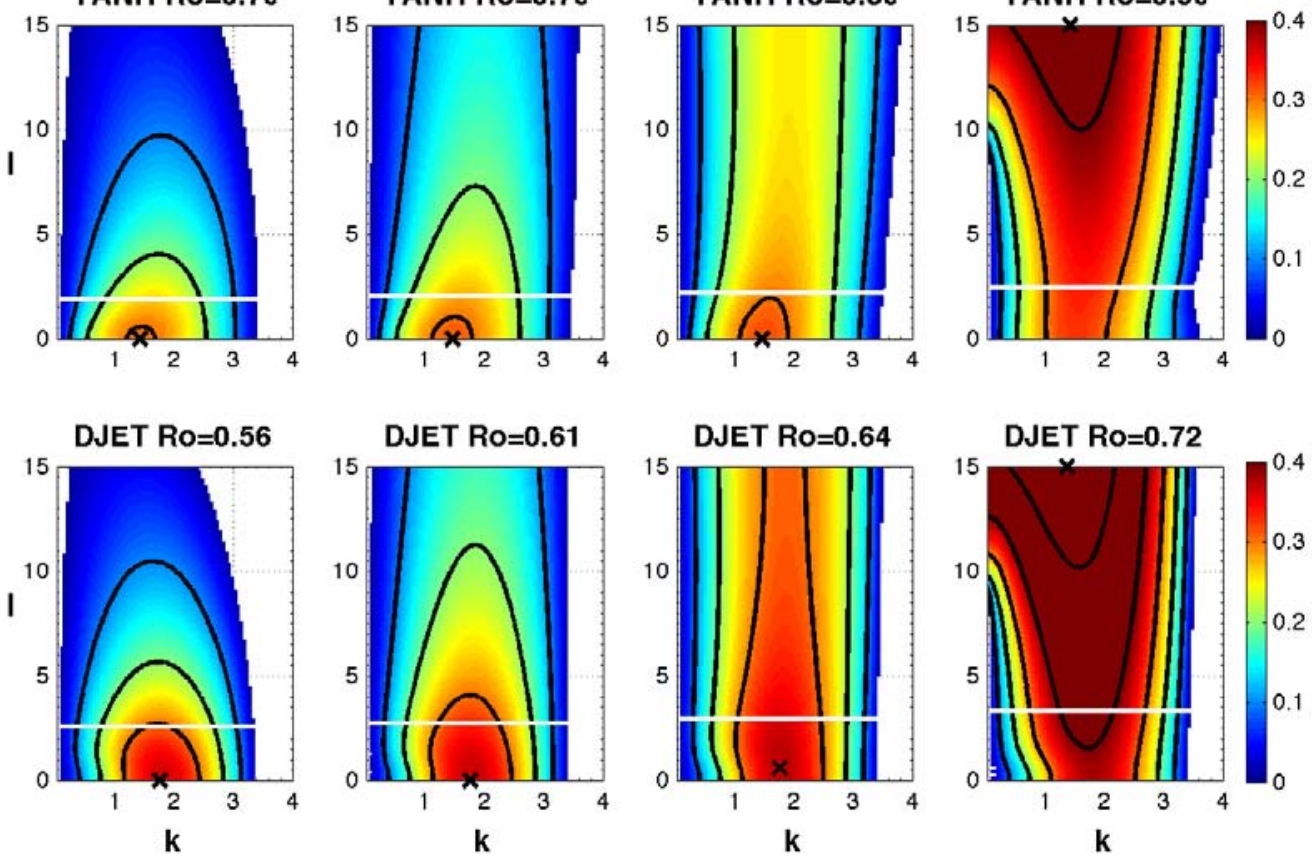

FiguRE 10 . The growth rate of the vertically symmetric mode $(\omega=0)$ in the EADY, TANH, and DJET flows in a $(k, l)$ space with increasing $R o$. The $R o$ in the second column are the onset criteria for CI. In the first three columns, the AI1 is dominant in the shown $(k, l)$ region. As $P V$ becomes strongly negative in the fourth column, the CI becomes the dominant mode in this $(k, l)$ region. The minimum value for the contours is 0.1 , and their interval is 0.1 . The $(k, l)$ for the maximum growth rates for each $R o$ are denoted by the cross symbols in the subplots. The white horizontal line in the TANH and DJET subplots denotes the lower limit of $l$ above which the perturbations are safely calculated with the "wide jet approximation".

The ageostrophic instability in the EADY flow has been interpreted as a coupling between an edge wave and an inertia-gravity wave that occurs with an inertia critical layer (Nakamura 1988; Plougonven et al. 2005), and hence we categorize it as an AI2 type of mode. In an interior shear flow with positive $P V$ and without a $P V$ singularity or edges, there are two types of shear-modified neutral wave: a balanced shear wave that exists due to non-zero $P V$ gradient and an inertia-gravity wave. The coupling of two 
waves in any combination could lead to an instability (i.e., a "resonant" coincidence of two neutral $\omega$ values leading a $\pm \sigma \neq 0$ pair without diffusion). For instance, the classic Eady baroclinic instability can be described as a coupling of two edge waves (a special type of balanced shear wave). We name the AI2 that couples balanced shear waves and inertia-gravity waves as a BG mode ("B" refers to balanced shear waves, and "G" refers to inertia-gravity waves), and the AI2 that couples two inertia-gravity waves as a GG mode.

\subsection{AI2 of BG type}

The EADY ageostropic instability is of the BG type, but special due to its dependence on the vertical boundaries. For a $f$-plane vertical shear $\bar{U}(z)$, the horizontal gradient of $P V$ is

$$
P V_{y}=-R o \bar{U}_{z z}
$$

a non-zero $P V_{y}\left(\bar{U}_{z z}\right)$ supports the existence of a balanced shear wave (Rossby wave). The EADY flow has a zero interior $P V_{y}$ hence there are no interior shear waves, but the horizontal buoyancy gradient (proportional to $\bar{U}_{z}$ ) at the vertical boundaries supports the existence of edge waves. Therefore in the EADY flow, the vertical boundary is essential for the occurrence of an unstable BG mode.

If we consider a flow profile transformation from EADY to TANH, $\bar{U}_{z}$ gradually vanishes at the boundaries and $P V_{y}$ begins to appear in the interior. The classic BCI could still exist due to the appearance of interior $P V_{y}$ with both signs. What about the AI2? An experiment is designed to examine the change of the ageostrophic instability in the transformation. We study a series of hyperbolic tangent mean flows,

$$
\bar{U}(z)=\frac{1}{a} \tanh (a z),
$$

in a fixed domain $[-0.50 .5]$. The mean flow has a parameter $a$ to control its shape and $\bar{U}_{z}$ values at the boundaries. All the mean flows are normalized to have the same $\bar{U}_{z}$ $=1$ at $z=0$. When $a \rightarrow 0$, the flow becomes an EADY flow and $\bar{U}_{z}=1$ everywhere. As $a$ increases, $\bar{U}_{z}$ vanishes at the boundaries, and the interior $P V_{y}\left(\bar{U}_{z z}\right)$ increases. The growth rate of the BCI mode does not vary significantly with $a$, while that of the BG mode changes dramatically. Figure 11(a, b) show the growth rates of the BG mode for the mean flows with $\bar{U}_{z}=1$ and $\bar{U}_{z}=0.9$ at the boundaries, respectively. The BG mode splits in $k$ when $\bar{U}_{z}$ decreases; the right part occurs towards larger $k$ while the left persists in the same $k$ range. The growth rates of both parts decrease dramatically with decreasing $\bar{U}_{z}$. The maximum growth rates for the BG mode in $k<2.5$ and $k>$ 2.5 versus $\bar{U}_{z}$ are shown in Fig. 11(c). In both $k$ ranges, the BG mode vanishes when $\bar{U}_{z}$ decreases, with the large- $k$ BG mode vanishing more quickly. When $\bar{U}_{z}$ vanishes, the edge wave no longer exists, therefore the EADY BG mode disappears. However, different from the BCI, no other BG type AI2 mode arises with increasing interior $P V_{y}$ in the hyperbolic tangent flows, even for a large value of $a$.

Through a coupling of an interior shear wave and an inertia-gravity wave, a BG mode is found in the DJET flow. Figure 12 shows the perturbation steamfunction pattern for the BG mode in the DJET flow. The mode appears with an inertia critical layer and clearly consists of two components. One component is confined near the ICL and it is highly unbalanced; this is the inertia-gravity wave component. The balanced component, an interior shear wave, is in the mean shear region. It is supported by the outer $P V_{y}$ in the velocity roll-off region that does not exist in the TANH flow. The structure of this BG mode is very similar to that in the EADY flow (Fig. 7 in Molemaker et al. (2005)), except for the vertical location of the shear wave. Different from the EADY BG 

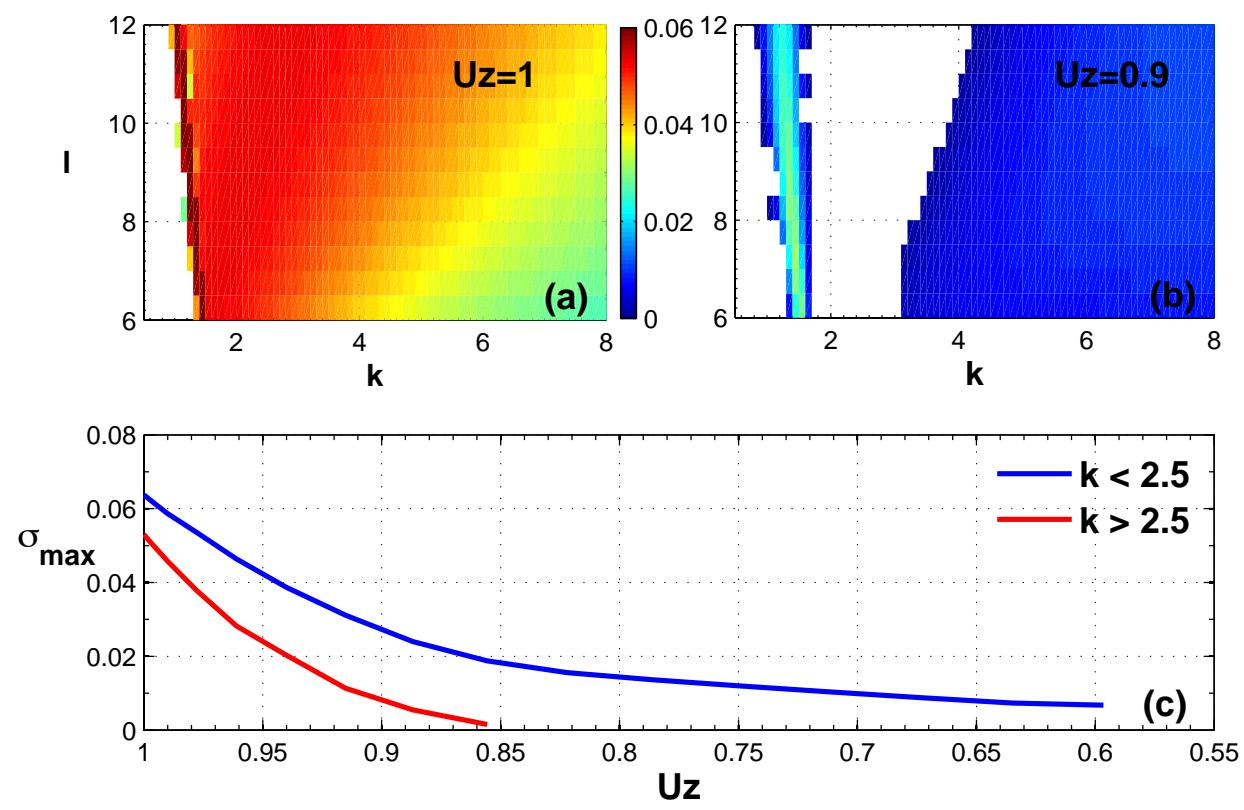

Figure 11. The BG AI2 mode vanishes in the EADY flow as $U_{z}=U_{z}( \pm 0.5)$ decreases at the boundaries due to the increase of $a$ in (5.2). The upper plots (a, b) demonstrate the growth rate $\sigma(k, l)$ of the BG mode for $\bar{U}_{z}=1(a=0)$ and $\bar{U}_{z}=0.9(a=0.65)$, respectively, with the same color scale. The lower plot (c) shows the maximum growth rates in a $(k, l)$ space versus the value of $\bar{U}_{z}$ at the boundaries for $k<2.5$ (blue) and $k>2.5$ (red), respectively. Ro is 1 for all the results. The vertically symmetric AI1 mode with $\omega=0$ is not included here.

mode, this interior BG mode only occurs at small and intermediate $k$. Like AI1, the BG type AI2 in the DJET flow comes from a continuous transformation of a BCI mode with vertically asymmetric structure and non-zero frequency. The growth rates of this vertically asymmetric mode in a $(k, l)$ space for $R o=0.01$ and $R o=1$ are shown in Fig. $13(\mathrm{a})$ and $13(\mathrm{~b})$. At $R o=0.01$, the BCI mode appears near $l=0$ with a weak growth rate. The mode is balanced and the perturbation energy conversion is from potential to kinetic. As $R o$ increases, this mode expands to intermediate $k$ and $l$ with an increasing growth rate; the inertia-gravity wave begins to couple with the shear flow, and an ICL starts to appear at some height in the perturbation fields for intermediate $(k, l)$. At $R o$ $=1$, the mode has expanded to large $l$ and it is unbalanced by a coupling with inertiagravity waves for all the intermediate and large $k$ regions. The main energy source for this interior BG mode is the mean kinetic energy, and the mode converts energy from kinetic to potential at intermediate and large $l$. Figure 13(c) shows the growth rate versus $R o$ for three particular pairs of $(k, l)$ values. The BG mode starts to occur in the neighborhood of $A-S=0(R o=0.43)$ and below the $R o$ onset of CI $(R o=0.61)$, hence can also be categorized as an AAI. The BG mode in both the EADY and DJET flow has a symmetric reflected companion with the same growth rate and an opposite frequency. For a BG mode with an eigenvalue $\tilde{\sigma}$ and eigenfunctions $\{\hat{u}, \hat{v}, \hat{w}, \hat{p}, \hat{b}\}(z)$, its companion mode has an eigenvalue $\tilde{\sigma}^{*}$ and the eigenfunctions are $i\left\{\hat{u}^{*}, \hat{v}^{*}, \hat{w}^{*},-\hat{p}^{*}, \hat{b}^{*}\right\}(-z)$.

We searched extensively in a $(k, l, R o)$ space in the TANH flow and did not find an unstable BG mode. The BG mode disappears when the EADY flow is transformed to the TANH flow, as the boundary wave vanishes. While the TANH flow is changed to 

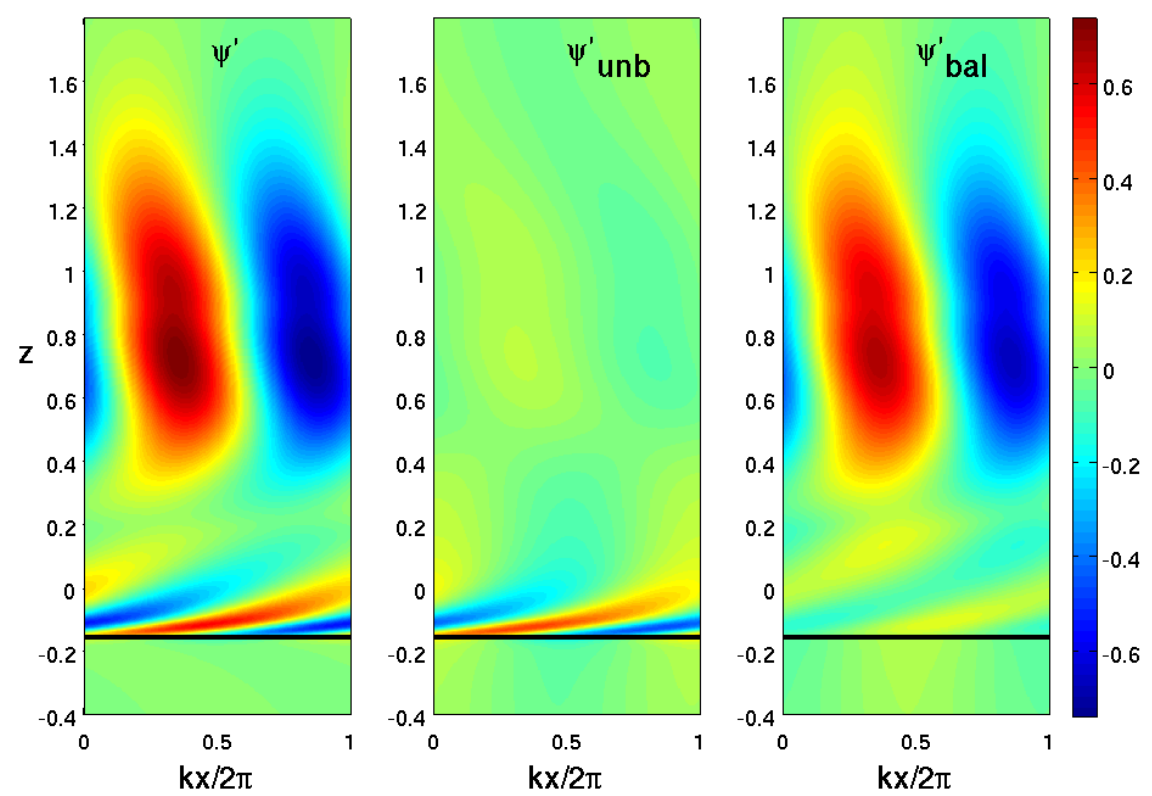

FiguRE 12. The perturbation streamfunction patterns of a BG mode (full, unbalanced, and balanced components, with the same color scale) in the DJET flow for $k=1.8, l=3.5$, and $R o=1$; its frequency $\omega=0.55$ and growth rate $\sigma=0.05$. The black horizontal line shows the inertia critical layer.

the DJET flow by vanishing the velocity towards boundary, a shear wave develops by the "outer" velocity roll-off, and therefore the BG mode occurs. In all the BG modes that we examine, the G mode does not couple with the "center" shear where $\overline{P V}_{y}$ is the maximum, but only couples with the "outer" or boundary shear where the velocity is large.

\subsection{AI2 of GG type}

We also find a type of AI2 that can be interpreted as a coupling of two inertia-gravity waves; we call it as a GG mode. Different from the AI2 of BG type, the GG modes occurs in all the three types of mean flow we examine. As mentioned in Sec. 4, the highly unbalanced zero-frequency instability that occurs at $R o \geqslant 0.8$ and intermediate $k$ is a GG mode. The characteristics of this mode in the TANH flow is shown in Fig. 14. It appears at intermediate $k$, for both small and large $l$. The mode is mostly hydrostatic and starts to appear around $R o \sim 0.8$, well above the onset $R o$ of AAI $(R o=0.53)$ but far below that of KHI $(R o=1.51)$. It is highly unbalanced for intermediate $R o$, including all the velocity perturbations $u^{\prime}, v^{\prime}$ and $w^{\prime}$. An unstable GG mode grows by drawing energy from the available potential energy of the mean state, and the instability transfers the perturbation kinetic to the mean kinetic energy. A GG mode has two inertia critical layers (shown by the horizontal black lines in the eigenmode plots), with two inertia-gravity wave components confined near them. The sheared, component inertia-gravity waves are Doppler-shifted to have the same frequency by the oppositely directed mean flow aloft and below.

The AI2 mode of the GG type in the DJET flow (not shown) has very similar characteristics to the GG mode in the TANH flow. There is a pair of inertia critical layers appearing 

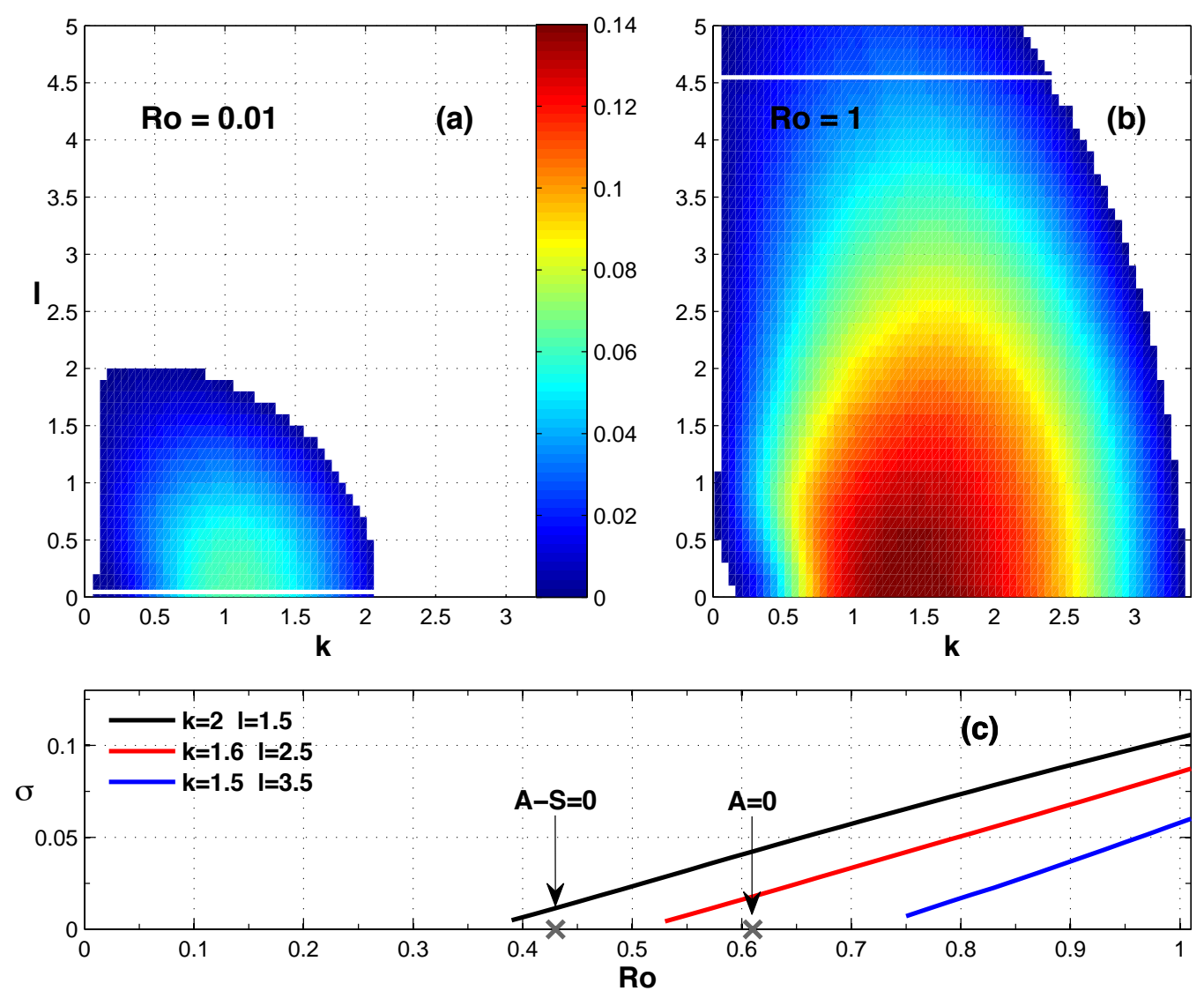

FIGURE 13 . The growth rate of the vertically asymmetric mode $(\omega \neq 0)$ in the DJET flow in a $(k, l)$ space is shown for $R o=0.01$ (a) and $R o=1$ (b). (c) shows the growth rate versus $R o$ for three particular $(k, l)$ pairs. The black represents $k=2, l=1.5$, the red represents $k=1.6$, $l=2.5$, and the blue represents $k=1.5, l=3.5$. The cross symbols denote the $R o$ where $\bar{A}-\bar{S}$ $=0$ and $\bar{A}=0$. The white horizontal line in each $R o$ subplot denotes the lower limit of $l$ above which the perturbations are safely calculated with the "wide jet approximation".

in the mode structure; the confined inertia-gravity wave components are Doppler-shifted by the local mean flow with opposite signs. The Ro regime of its occurrence is also between AAI and KHI.

All the AI2 modes (including BG and GG) have inertia critical layers and hence are associated with the coupling of inertia-gravity waves to the mean shear. Because of the continuous transformation of mode characteristics in ageostrophic instability, rather than arising with a distinct separation in $(k, l)$ from other types of instability, there is not unique definition of a representative growth rate for the two types of AI2 mode at a fixed $R o$. In this section, the growth rate dependence on $R o$ is shown for several $(k, l)$ pairs in Fig. 13(c), which indicate the relevance of the $A-S$ condition as a neighborhood for onset of BG type AI2; therefore the BG mode can be an AAI in type. The GG mode arises away from $A-S=0$ in Fig. 14(c), hence is not associated with AAI. 

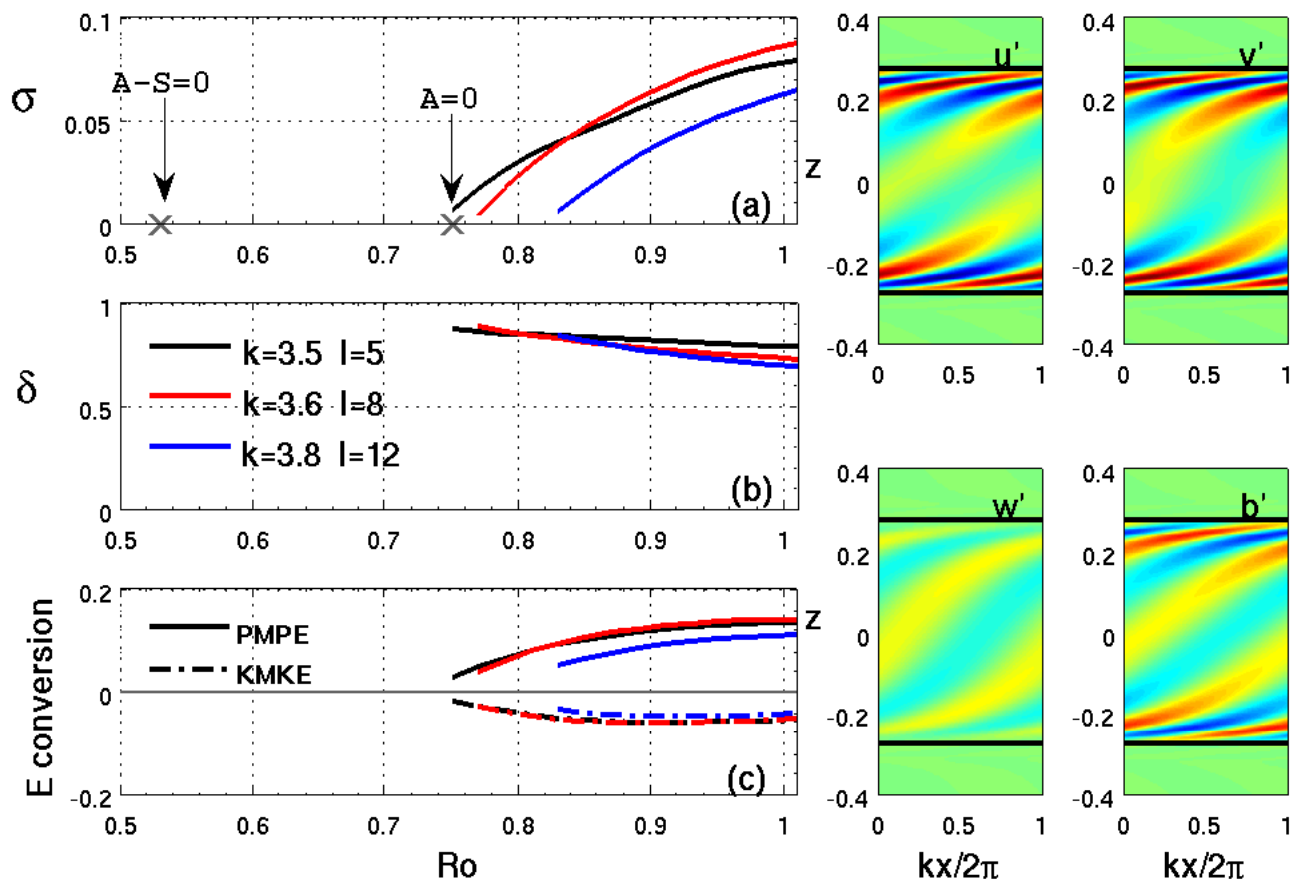

FiguRE 14. Diagnostics and eigenmode patterns for the second type of ageostrophic instability (AI2) of the GG type in the TANH flow. The left plots are (a) the growth rate, (b) the degree of unbalance $\delta$, and (c) the energy conversion from mean potential energy (PMPE, solid lines) and mean kinetic energy (KMKE, dash-dot lines) varying with $R o$, respectively. Three particular $(k, l)$ groups are shown, the black represents $k=3.5, l=5$, the red represents $k=3.6, l=8$, and the blue represents $k=3.8, l=12$ in (a), (b) and (c). The right plots are the normalized eigenmode patterns $\left(u^{\prime}, v^{\prime}, w^{\prime}, b^{\prime}\right)$ of a GG mode $(\sigma=0.02$ and $\omega=0)$ for $k=3.8, l=12$, and $R o=0.85$, all with the same color scale. Red represents positive, and blue represents negative. The inertia critical layers are shown by the black horizontal lines in the mode pattern plots. The cross symbols in (a) denote the $R o$ where $\bar{A}-\bar{S}=0$ and $\bar{A}=0$.

By checking the validity of the results in this section with the "wide jet approximation", we find that the BG mode in the EADY to TANH transition (Fig. 11) and the AI2 of GG type in the TANH flow (Fig. 14) are reliably calculated. However, the BG mode in the DJET flow is not safely calculated for intermediate and large $R o$, including the results shown in Fig. 12 and Fig. 13(b) and (c). The BG mode result in the DJET flow shows a possible type of ageostrophic instability that couples a shear wave and a gravity wave in a general, interior vertical shear other than the EADY flow; therefore, we choose to show it here despite its uncertainty. As mentioned in Sec. 2, further investigation by solving a $2 \mathrm{D}$ eigenvalue problem in a $y$-bounded domain could obtain an accurate answer for this BG mode, but it is not feasible with our current computational tool; therefore, a revisit of this problem is needed to confirm the existence of a BG mode in a general, interior vertical shear flow.

\section{Summary and discussion}

We solve the linear instability problem for an interior, baroclinic shear flow $\bar{U}(z)$ in a 3D, rotating, continuously stratified fluid. Besides a revisitation of the EADY flow, two 
new types of odd-symmetric mean flows are examined: TANH (hyperbolic tangent flow) and DJET (double-jet flow). We find two types of baroclinic, ageostrophic instability, both of which are mostly hydrostatic with a small aspect ratio $\lambda$. They do not exist in quasigeostrophic or higher-order Balanced Equations. They start to occur at finite Ro, and their growth rate increases with increasing $R o$. The first type of ageostrophic instability (AI1) comes from a continuous transformation of mode properties and expansion in $(k, l)$ space between the classic baroclinic instability $(\mathrm{BCI})$ and centrifugal instability (CI). It occurs between the Ro regimes for $\mathrm{BCI}$ and $\mathrm{CI}$ in a $(k, l)$ range that is far from the familiar regions for the occurrence of BCI (near $l=0$ ) and CI (near $k=0$ ). The AI1 mode has a very small unbalanced component for small $k$, and it becomes moderately unbalanced as $k$ increases. Unlike the BCI and CI, the main energy source for AI1 is the mean kinetic energy, and the unstable perturbation transfers energy to the mean potential energy. The AI1 mode is found in all three mean flows, except that it appears in a narrower band of $k$ in the EADY flow. The second type of ageostrophic instability (AI2) is associated with inertia critical layers and related to a coupling of inertia-gravity waves to the mean shear. The coupling is either between a balanced shear wave and an inertia-gravity wave (BG type), or between two inertia-gravity waves (GG type). The EADY BG mode is caused by a coupling between an edge wave (a special boundary shear wave) and an inertia-gravity wave. In a transformation from a EADY to TANH flow, the BG mode in the EADY flow disappears with vanishing $\bar{U}_{z}$ at boundaries (proportional to the horizontal buoyancy gradient), while no other BG mode that is associated with an interior potential vorticity gradient arises with increasing interior $\bar{U}_{z z}$ (proportional to $P V_{y}$ ) in the TANH flow. With a roll-off velocity towards boundary, the BG type AI2 mode is found in the DJET flow, as a coupling between an interior shear wave and an inertia-gravity wave. It appears with an inertia critical layer, and both its balanced and unbalanced components are clearly identified in the perturbation patterns. In TANH and DJET flows, we find AI2 modes of a GG type. The GG mode has two inertia-gravity wave components that are Doppler-shifted by oppositely directed local mean flows and confined near the inertia critical layers. The main energy source for an AI2 BG mode is the mean kinetic energy, while that for an AI2 GG mode is the mean potential energy. Both AI1 and AI2 are unbalanced instabilities, with the growth rate of AI2 generally smaller than AI1. Within the $(k, l, R o)$ regime we examined, the existence of the ageostrophic instabilities (AI1, AI2 and CI) in the three mean flows is summarized in Table 2.

We have also examined the ageostrophic instabilities in an even $z$-symmetric interior one-jet with $\bar{U}=0.5 / \cosh ^{2}(3.8 z)$ ("Bickley" jet), and find AI1, and AI2 of both BG and GG types in this mean flow. Due to the non-zero $z$-average of this mean flow, the AI1 mode has a non-zero frequency, but all the other characteristics (e.g., continuous transformation in $R o$ and $(k, l)$ space, energetics, degree of balance) are very similar to the above odd-symmetric flows. The BG mode appears in pairs symmetric about $z=0$, sitting on both sides off the jet center, hence it has two inertia critical layers (a "double" BG mode). The GG mode also appears in pairs, and therefore has four inertia critical layers in the mode pattern (a "double" GG mode). Though with some minor difference due to the $z$-symmetry, both AI1 and AI2 behave quite the same in the interior one-jet as the odd-symmetric flows, therefore we do not show detailed results for the interior one-jet here. By the study of both even-symmetric and odd-symmetric mean flows, we find AI1 and AI2 are general behaviors in an interior $U(z)$, with the one exception that no BG mode is found in the TANH flow even after an extensive search.

Both AI1 and AI2 of the BG type occur in the neighborhood of $A-S=0$ in the interior $\bar{U}(z)$ flows, hence are in the AAI category. As other AAI modes in the previous literature, they do not have a sharp onset criterion (e.g., unlike CI). The AI2 of GG type 
TABLE 2. A summary for the existence of the ageostrophic instabilities in the $(k, l, R o)$ regime examined in our study for the three types of mean flows.

\begin{tabular}{lccc}
\hline \hline & EADY & TANH & DJET \\
\hline AI1 & $\checkmark$ & $\checkmark$ & $\checkmark$ \\
\hline AI2 BG & $\checkmark$ & $\times$ & $\checkmark$ \\
\hline AI2 GG & $\times$ & $\checkmark$ & $\checkmark$ \\
\hline CI & $\checkmark$ & $\checkmark$ & $\checkmark$ \\
\hline
\end{tabular}

occurs for a $R o$ value beyond the $A-S$ condition, and therefore it is not likely to be a type of AAI. The characteristics of the AI1 and AI2 modes are not the same, and they are not the same as other AAI modes previously found in other flows (Sec. 1), including the EADY flow. This indicates that AAI is more a family of unstable mode types rather than a single unique type. AI1 does not have evident inertia-gravity wave characteristics (radiation or inertia critical layers), but AI2 does, indicating that not all the unbalanced instabilities and loss of balance in a continuously stratified flow are related to an energy transfer to inertia-gravity waves. Unlike the ageostrophic instability in the EADY flow, both AI1 and AI2 do not require vertical boundaries. Both instabilities are absent in the quasigeostrophic limit $(R o \rightarrow 0)$, are distinct from BCI, and start to occur well below the Ro criteria for CI and KHI.

The literature and our study have shown plenty of distinct examples of ageostrophic instabilities: some occur with boundaries, singular potential vorticity gradient, surface front, or other special "edges", and some occur in interior flows; some have clear onset conditions, like $\mathrm{CI}$ or $\mathrm{AI}$ in shallow water, and some do not have a sharp criterion such as AAI; some are associated with inertia-gravity waves, and some are not. The examined ageostrophic instabilities have a variety of physical mechanisms and energetics, and are possibly related to different theoretical criteria. The general story for the ageostrophic instability is not complete: it has been better developed with purely barotropic or baroclinic mean shears, but due to the computational limitation, an extensive, high resolution study on a $2 \mathrm{D}$ non-seperable shear is not finished yet. Nevertheless, we now know that ageostrophic instability is common in flows with intermediate $R o$ values, and this process enables a possible local route for the loss of balance and forward energy cascade in the interior ocean and atmosphere.

We thank anonymous reviewers for their constructive recalcitrance. This research is partially supported by the National Science Foundation (OCE-1049134) and Office of Naval Research (N00014-10-1-0484).

\section{Appendix A. Non-dimensional translation and parameter comparison with Stone $(1966,1970)$}

This appendix describes the non-dimensional translation between the model in Stone $(1966,1970)$ and our study, with a detailed parameter comparison listed in Table 3. In 
Stone $(1966,1970)$, the time is scaled by rotation rate $t \rightarrow f^{-1}$, and the horizontal length is scaled as $L \rightarrow U_{0} / f$. With this non-dimensionalization, $R o=U_{0} / f L$ has a constant value 1 , and $B u=(R o / F r)^{2}=1 / F r^{2}=R i$. In our study, the time is scaled as $t \rightarrow L / U_{0}$, and we have a fixed value for $B u$; and therefore in our numerical experiments $B u=1$, $R o=F r$, and $1 / F r^{2}=R i$.

We have mentioned in Sec. 2 that the parameters $R o, B u$ and $\lambda$ are not independent to each other. In the following part, we show that for any set of $(B u, R o, \lambda, k, l)$, there is an equivalent set of $\left(B u^{*}, R o^{*}, \lambda^{*}, k^{*}, l^{*}\right)$ that yields the same eigenvalue problem as (2.5). With the definition $B u=\left(\frac{N H}{f L}\right)^{2}$, we vary the horizontal length scale $L^{*}=L / \sqrt{\alpha}$ to let $B u^{*}=\alpha B u$, and keep the vertical length scale and the velocity scales the same. The variables are then scaled as

$$
\begin{gathered}
\left(x^{*}, y^{*}\right) \rightarrow L^{*}, \quad z^{*} \rightarrow H, \quad\left(u^{*}, v^{*}\right) \rightarrow U_{0}, \quad t^{*} \rightarrow \frac{L^{*}}{U_{0}}, \\
w^{*} \rightarrow W_{0}=\frac{f U_{0}^{2}}{N^{2} H}, \quad p^{*} \rightarrow f U_{0} \rho_{0} L^{*}, \quad b^{*} \rightarrow \frac{f U_{0} L^{*}}{H} .
\end{gathered}
$$

Here we use $a^{*}$ notation to represent the new parameters and variables. With the new non-dimensionalization, we have

$$
\left(B u^{*}, R o^{*}, \lambda^{*}, k^{*}, l^{*}\right)=\left(\alpha B u, \sqrt{\alpha} R o, \sqrt{\alpha} \lambda, \frac{k}{\sqrt{\alpha}}, \frac{l}{\sqrt{\alpha}}\right),
$$

and

$$
\left(\tilde{\sigma}^{*}, \hat{u}^{*}, \hat{v}^{*}, \hat{w}^{*}, \hat{p}^{*}, \hat{b}^{*}\right)=\left(\frac{\tilde{\sigma}}{\sqrt{\alpha}}, \hat{u}, \hat{v}, \hat{w}, \sqrt{\alpha} \hat{p}, \sqrt{\alpha} \hat{b}\right) .
$$

Substituting the above relation into (2.5), we reach

$$
\left\{\begin{aligned}
R o^{*}\left(\tilde{\sigma}^{*} \hat{u}^{*}+i k^{*} \bar{U} \hat{u}^{*}+\frac{R o^{*}}{B u^{*}} \bar{U}_{z} \hat{w}^{*}\right)-\hat{v}^{*}+i k^{*} \hat{p}^{*} & =0 \\
R o^{*}\left(\tilde{\sigma}^{*} \hat{v}^{*}+i k^{*} \bar{U} \hat{v}^{*}\right)+\hat{u}^{*}+i l^{*} \hat{p}^{*} & =0 \\
\lambda^{* 2} \frac{R o^{* 2}}{B u^{*}}\left(\tilde{\sigma}^{*} \hat{w}^{*}+i k^{*} \bar{U} \hat{w}^{*}\right)-\hat{b}^{*}+\frac{\partial \hat{p}^{*}}{\partial z} & =0 \\
i k^{*} \hat{u}^{*}+i l^{*} \hat{v}^{*}+\frac{R o^{*}}{B u^{*}} \frac{\partial \hat{w}^{*}}{\partial z} & =0 \\
\tilde{\sigma}^{*} \hat{b}^{*}+i k^{*} \bar{U} \hat{b}^{*}-\bar{U}_{z} \hat{v}^{*}+\hat{w}^{*} & =0 .
\end{aligned}\right.
$$

The new eigenvalue problem (A 3) has the same form as (2.5). Therefore, to solve the problem (2.5) with any set of $(B u, R o, \lambda, k, l)$, is equivalent to solve it with $(B u=$ $1, R o / \sqrt{B u}, \lambda / \sqrt{B u}, \sqrt{B u} k, \sqrt{B u} l)$; and the eigenvalue and eigenfunctions follow the translation rules (A 2).

According to the translation rules (A 1) and (A 2), Table 3 gives the equivalent parameter settings for the EADY flow in Stone (1970) and in our study.

\section{REFERENCES}

Boss, E., Paldor, N. \& Thompson, L. 1996 Stability of a potential vorticity front: from quasi-geostrophy to shallow water. J. Fluid Mech. 315, 65-84.

Bouchut, F., Ribstein, B. \& Zeitlin, V. 2011 Inertial, barotropic, and baroclinic instabilities of the Bickley jet in two-layer rotating shallow water model. Phys. Fluids 23, 126601.

Chandrasekhar, S. 1961 Hydrodynamic and Hydromagnetic Stability. Cambridge University Press. 
TABLE 3. Equivalent parameter settings in Stone (1970) (left) and our study (right) for the EADY flow. Note that for Stone (1970), Ro is constant as 1, and in our study, Bu is constant as 1 .

\begin{tabular}{ll}
\hline \hline Stone $(1970)(R o=1)$ & Our study $(B u=1)$ \\
\hline$R i=2,(k, l)=(1,1)$ & $R o=0.71,(k, l)=(1.41,1.41)$ \\
\hline$R i=1,(k, l)=(1,1)$ & $R o=1,(k, l)=(1,1)$ \\
\hline$R i=0.92,(k, l)=(1,1)$ & $R o=1.04,(k, l)=(0.96,0.96)$ \\
\hline$R i=0.5,(k, l)=(1,1)$ & $R o=1.41,(k, l)=(0.71,0.71)$ \\
\hline$R i=0.1,(k, l)=(1,1)$ & $R o=3.16,(k, l)=(0.32,0.32)$ \\
\hline
\end{tabular}

Charney, J. G. 1947 The dynamics of long waves in a baroclinic westerly current. J. Atmos. Sci. 4, 136-162.

Charney, J. G. 1962 Integration of the Primitive and Balanced Equations. Proc. Int. Symp. Numerical Weather Prediction pp. 131-152.

Charney, J. G. 1971 Geostrophic turbulence. J. Atmos. Sci. 28, 1087-1094.

Drazin, P. G. \& Reid, W. H. 1981 Hydrodynamic Stability. Cambridge University Press.

Dritschel, D. G. \& VAnneste, J. 2006 Instability of a shallow-water potential-vorticity front. J. Fluid Mech. 561, 237-254.

EADY, E. T. 1949 Long waves and cyclone waves. Tellus 1, 33-52.

Gent, P. R. \& McWilliams, J. C. 1982 Intermediate model solutions to the Lorenz Equations: strange attractors and other phenomena. J. Atmos. Sci. 39, 3-13.

Griffiths, R. W., Killworth, P. D. \& Stern, M. E. 1982 Ageostrophic instability of ocean currents. J. Fluid Mech. 117, 343-377.

Gula, J., Plougonven, R. \& Zeitlin, V. 2009 Ageostrophic instabilities of fronts in a channel in a stratified rotating fluid. J. Fluid Mech. 627, 485-507.

Gula, J. \& Zeitlin, V. 2010 Instabilities of buoyancy-driven coastal currents and their nonlinear evolution in the two-layer rotating shallow water model. Part 1. Passive lower layer. J. Fluid Mech. 659, 69-93.

Gula, J., Zeitlin, V. \& Bouchut, F. 2010 Instabilities of buoyancy-driven coastal currents and their nonlinear evolution in the two-layer rotating shallow water model. Part 2. Active lower layer. J. Fluid Mech. 665, 209-237.

Hayashi, Y. Y. \& Young, W. R. 1987 Stable and unstable shear modes of rotating parallel flows in shallow waters. J. Fluid Mech. 184, 477-504.

Hoskins, B. J. 1974 The role of potential vorticity in symmetric stability and instability. Q. J. Roy. Meteor. Soc. 100, 480-482.

Jones, W. L. 1967 Propagation of internal gravity waves in fluids with shear flow and rotation. J. Fluid Mech. 30, 439-448.

Killworth, P., Paldor, N. \& Stern, M. 1984 Wave propagation and growth on a surface front in a two-layer geostrophic current. J. Mar. Res. 42, 761-785.

Kubokawa, A. 1985 Instability of a geostrophic front and its energetics. Geophys. Astro. Fluid 33, 323-357.

Kubokawa, A. 1986 Instability caused by the coalescence of two modes of a one-layer coastal current with a surface front. J. Oceanogr. 42, 373-380.

LEITH, C. E. 1980 Nonlinear normal mode initialization and quasi-geostrophic theory. J. Atmos. Sci. 37, 958-968.

LoREnZ, E. 1960 Energy and numerical weather prediction. Tellus 12, 364-373. 
Lorenz, E. N. 1980 Attractor sets and quasi-geostrophic equilibrium. J. Atmos. Sci. 37, 16851699.

Lorenz, Edward N. 1986 On the existence of a slow manifold. J. Atmos. Sci. 43, 1547-1558.

Lorenz, Edward N. \& Krishnamurthy, V. 1987 On the nonexistence of a slow manifold. $J$. Atmos. Sci. 44, 2940-2950.

McWilliams, J. C., Molemaker, M. J. \& Yavneh, I. 2004 Ageostrophic, anticyclonic instability of a geostrophic, barotropic boundary current. Phys. Fluids 16, 3720-3725.

MCWilliams, J. C. \& Yavneh, I. 1998 Fluctuation growth and instability associated with a singularity of the Balance Equations. Phys. Fluids 10, 2587-2596.

McWilliams, J. C., Yavneh, I., Cullen, M. J. P. \& Gent, P. R. 1998 The breakdown of large-scale flows in rotating, stratified fluids. Phys. Fluids 10, 3178-3184.

Ménesguen, C., McWilliams, J. C. \& Molemaker, M. J. 2012 Ageostrophic instability in a rotating stratified interior jet. J. Fluid Mech. 711, 599-619.

Miles, J. W. 1961 On the stability of heterogeneous shear flows. J. Fluid Mech. 10, 496-508.

Molemaker, M. J., McWilliams, J. C. \& YavneH, I. 2001 Instability and equilibration of centrifugally stable stratified Taylor-Couette flow. Phys. Rev. Lett. 86, 5270-5273.

Molemaker, M. J., McWilliams, J. C. \& Yavneh, I. 2005 Baroclinic instability and loss of balance. J. Phys. Oceanogr. 35, 1505-1517.

NAKAmURA, N. 1988 Scale selection of baroclinic instability-effects of stratification and nongeostrophy. J. Atmos. Sci. 45, 3253-3268.

Ooyama, K. 1966 On the stability of the baroclinic circular vortex: A sufficient condition for instability. J. Atmos. Sci. 23, 43-53.

PALdor, N. 1983 Linear stability and stable modes of geostrophic fronts. Geophys. Astro. Fluid 24, 299-326.

Pedlosky, J. 1979 Geophysical Fluid Dynamics. New York and Berlin, Springer-Verlag.

Phillips, N. A. 1954 Energy transformations and meridional circulations associated with simple baroclinic waves in a two-level, quasi-geostrophic model. Tellus 6, 273-286.

Plougonven, R., Muraki, D. J. \& Snyder, C. 2005 A baroclinic instability that couples balanced motions and gravity waves. J. Atmos. Sci. 62, 1545-1559.

RIPA, P. 1983 General stability conditions for zonal flows in a one-layer model on the beta-plane or the sphere. J. Fluid Mech. 126, 463-489.

SAKAI, S. 1989 Rossby-Kelvin instability: a new type of ageostrophic instability caused by a resonance between Rossby waves and gravity waves. J. Fluid Mech. 202, 149-176.

Stone, P. H. 1966 On non-geostrophic baroclinic stability. J. Atmos. Sci. 23, 390-400.

Stone, P. H. 1970 On non-geostrophic baroclinic stability: Part II. J. Atmos. Sci. 27, 721-726.

Sutyrin, G. G. 2007 Ageostrophic instabilities in a horizontally uniform baroclinic flow along a slope. J. Fluid Mech. 588, 463-473.

Sutyrin, G. G. 2008 Lack of balance in continuously stratified rotating flows. J. Fluid Mech. 615, 93.

VAnNeste, J. \& YAVNeh, I. 2007 Unbalanced instabilities of rapidly rotating stratified shear flows. J. Fluid Mech. 584, 373.

Wang, P., McWilliams, J. C. \& Kizner, Z. 2012 Ageostrophic instability in rotating shallow water. J. Fluid Mech. 712, 327-353.

WARN, T. 1997 Nonlinear balance and quasi-geostrophic sets. Atmos.-Ocean 35, 135-145.

Warn, T., Bokhove, O., Shepherd, T. G. \& Vallis, G. K. 1995 Rossby number expansions, slaving principles, and balance dynamics. Quart. J. Roy. Meteor. Soc. 121, 723-739.

YAMANAKA, M. D. 1985 Inertial oscillation and symmetric motion induced in an inertio-gravity wave critical layer. J. Meteor. Soc. Japan 63, 715-737.

Yavneh, I., McWilliams, J. C. \& Jeroen Molemaker, M. 2001 Non-axisymmetric instability of centrifugally stable stratified Taylor-Couette flow. J. Fluid Mech. 448, 1-21. 